\title{
"Pre-semantic" cognition revisited: Critical differences between semantic aphasia and semantic dementia
}

\author{
Elizabeth Jefferies ${ }^{\mathrm{a}, *}$, Timothy T. Rogers ${ }^{\mathrm{b}}$, Samantha Hopper ${ }^{\mathrm{c}}$, Matthew A. Lambon Ralph ${ }^{\mathrm{c}}$ \\ a Department of Psychology, University of York, York YO10 5DD, UK \\ ${ }^{\mathrm{b}}$ University of Wisconsin-Madison, Madison, USA \\ c University of Manchester, UK
}

\section{A R T I C L E I N F O}

\section{Article history:}

Received 5 January 2009

Received in revised form 8 September 2009

Accepted 13 September 2009

Available online 18 September 2009

\section{Keywords:}

Semantic cognition

Semantic dementia

Aphasia

Pre-semantic

Reading

Verb inflection

Spelling

Lexical decision

Object decision

Colour decision

Picture copying

\begin{abstract}
A B S T R A C T
Patients with semantic dementia show a specific pattern of impairment on both verbal and non-verbal "pre-semantic" tasks, e.g., reading aloud, past tense generation, spelling to dictation, lexical decision, object decision, colour decision and delayed picture copying. All seven tasks are characterised by poorer performance for items that are atypical of the domain and "regularisation errors" (irregular/atypical items are produced as if they were domain-typical). The emergence of this pattern across diverse tasks in the same patients indicates that semantic memory plays a key role in all of these types of "pre-semantic" processing. However, this claim remains controversial because semantically impaired patients sometimes fail to show an influence of regularity. This study demonstrates that (a) the location of brain damage and (b) the underlying nature of the semantic deficit affect the likelihood of observing the expected relationship between poor comprehension and regularity effects. We compared the effect of multimodal semantic impairment in the context of semantic dementia and stroke aphasia on the seven "pre-semantic" tasks listed above. In all of these tasks, the semantic aphasia patients were less sensitive to typicality than the semantic dementia patients, even though the two groups obtained comparable scores on semantic tests. The semantic aphasia group also made fewer regularisation errors and many more unrelated and perseverative responses. We propose that these group differences reflect the different locus for the semantic impairment in the two conditions: patients with semantic dementia have degraded semantic representations, whereas semantic aphasia patients show deregulated semantic cognition with concomitant executive deficits. These findings suggest a reinterpretation of single-case studies of comprehensionimpaired aphasic patients who fail to show the expected effect of regularity on "pre-semantic" tasks. Consequently, such cases do not demonstrate the independence of these tasks from semantic memory. c) 2009 Elsevier Ltd. All rights reserved.
\end{abstract}

\section{Introduction}

Patients with semantic dementia (SD) have a highly selective and progressive impairment of semantic memory associated with bilateral atrophy of the anterior temporal lobes (ATL; Hodges, Patterson, Oxbury, \& Funnell, 1992; Mummery et al., 2000; Snowden, Goulding, \& Neary, 1989). Other cognitive functions, including phonology, syntax, executive skills and episodic memory, remain relatively intact in this condition. Nevertheless, SD patients show a highly predictable pattern of breakdown on a number of tasks typically thought to be "pre-semantic", including reading single words aloud (Funnell, 1996; Patterson \& Hodges, 1992; Woollams, Lambon Ralph, Plaut, \& Patterson, 2007); spelling to dictation (Graham, Patterson, \& Hodges, 2000; Parkin,

\footnotetext{
* Corresponding author. Tel.: +44 01904 434368; fax: +44 01904433181.

E-mail address: ej514@york.ac.uk (E. Jefferies).
}

1993); producing the past tense form of verbs from the present tense (Cortese, Balota, Sergent-Marshall, Buckner, \& Gold, 2006; Patterson, Lambon Ralph, Hodges, \& McClelland, 2001); lexical decision (Moss, Tyler, Hodges, \& Patterson, 1995; Rogers, Lambon Ralph, Hodges, \& Patterson, 2004); immediate serial recall of short lists of words (Jefferies, Jones, Bateman, \& Lambon Ralph, 2004; Jefferies, Jones, Bateman, \& Lambon Ralph, 2005; Knott, Patterson, \& Hodges, 1997; Knott, Patterson, \& Hodges, 2000); object decision, i.e., deciding if line drawings represent real objects (Breedin, Saffran, \& Coslett, 1994; Hovius, Kellenbach, Graham, Hodges, \& Patterson, 2003; Rogers, Hodges, Ralph, \& Patterson, 2003; Rogers, Lambon Ralph, Hodges, et al., 2004) and copying drawings of objects after a brief delay (Bozeat et al., 2003; Lambon Ralph \& Howard, 2000).

In all of these tasks, the input tends to specify the required output and thus might conceivably drive a response without any support from the semantic system. For example, in reading aloud, the strong connection between orthography and phonol- 
ogy might be able to generate the correct output independently of the meaning of the word. Studies of patients with SD suggest this is not the case for all words. These patients are poor at reading items with atypical spellings that they no longer fully understand and often pronounce them as if they were more typical (i.e., show surface dyslexia with regularisation errors such as PINT rhymes with mint; Woollams et al., 2007). This pattern suggests that semantic memory plays a critical role in deriving phonology from orthography, especially for words with unsystematic (or "irregular") orthography-to-phonology mappings (Plaut, McClelland, Seidenberg, \& Patterson, 1996). A similar pattern has been observed for the other "pre-semantic" tasks listed above: SD patients show poorer comprehension of lower frequency items (Bozeat et al., 2000; Funnell, 1995) and in every task in which regularity/typicality and frequency have been orthogonally varied, studies have shown an interaction between these variables, with greater impairments observed for lower frequency items that are atypical of the domain being investigated (references above).

A recent study obtained strong evidence to suggest that the semantic memory impairment in SD is causally linked to deficits on all of these "pre-semantic" tasks, despite their disparate nature (Patterson et al., 2006). Fourteen SD patients were tested on six tasks: reading aloud, spelling to dictation, past tense generation, lexical decision, object decision and delayed picture copying. In all six tasks, every patient showed the predicted pattern of significant impairment on lower frequency irregular/atypical items; and across patients, all tasks showed a significant interaction between frequency/familiarity and typicality/regularity (the sole exception being delayed-copy drawing where familiarity was not manipulated). In addition, errors for the atypical items were largely regularisations or LARC errors (legitimate alternative rendering of components). Regularisation errors occurred when the most frequent transformation was over-applied and so these errors were more typical of the domain than the correct response (e.g., in past tense generation, creep $\rightarrow$ "creeped"). The LARC errors were similar, but this time an alternative plausible transformation was over-applied (e.g., peep $\rightarrow$ "pept" as in "crept"). Finally, in all six "pre-semantic" tests, accuracy on the irregular items was strongly predicted by the level of semantic impairment, suggesting that deficits on individual tasks are a consequence of a central semantic impairment rather than parallel damage to unrelated domainspecific mechanisms.

In a series of papers, Patterson and colleagues have put forward an explanation of the impact of semantic memory impairment on these "pre-semantic" tasks (Patterson et al., 2006; Plaut et al., 1996; Rogers, Lambon Ralph, Garrard, et al., 2004; Woollams et al., 2007). Regular items that are typical of their domain are supported sufficiently by domain-specific representations alone, without the need for the additional support which comes automatically from semantic memory (all real words and objects have an associated meaning). For example, the correspondences between orthography and phonology are sufficient for reading regular words. Domain-specific representations are less able to specify the correct transformation for atypical items, however, and as a consequence, the automatic input from semantic memory plays an important role in constraining correct production. Regularisation/LARC errors occur when domain-specific representations reflecting transformations that are typical of the domain come to dominate attempts to produce irregular targets in the absence of support from semantic memory. This theory has been implemented in computational models of reading aloud (Plaut et al., 1996), past tense generation (Joanisse \& Seidenberg, 1999) and delayed picture copying (Rogers, Lambon Ralph, Garrard, et al., 2004).

Despite the strength of the empirical and theoretical work reviewed above, the view that semantic memory plays a key role in "pre-semantic" processing remains controversial. This is because semantically impaired cases sometimes fail to show the expected influence of regularity in particular tasks. For example, there are case reports of patients with profound semantic impairment who can successfully read words with irregular pronunciations (Blazely, Coltheart, \& Casey, 2005; Cipolotti \& Warrington, 1995; Gerhand, 2001; Lambon Ralph, Ellis, \& Franklin, 1995). These dissociations, although rare, are traditionally thought of as critical within cognitive neuropsychology because they imply that semantic impairment and surface dyslexia are separable. By this view, SD patients who are surface dyslexic have two independent deficits affecting semantic memory and reading respectively. ${ }^{1}$

In this context, it is important to note that while the association between semantic impairment and surface dyslexia is extremely strong, especially in SD (Woollams et al., 2007), there can be notable deviations away from the expected pattern. First, there are important and stable individual differences in domain-specific efficiency that modulate the exact degree of semantic reliance in reading aloud (e.g., some individuals rely on semantic processes more or less than the group average; Dilkina, McClelland, \& Plaut, 2008; Woollams et al., 2007). This results in varying degrees of surface dyslexia in patients with SD. Secondly, there might be crucial differences between patients with different aetiologies of brain damage. Although the relationship between semantic impairment and surface dyslexia is clear in SD, it may be weaker or even non-existent in other patient groups. We say "may" because there are very few studies investigating this link in other semantic syndromes. A few studies have shown that patients who have semantic impairment resulting from Alzheimer's disease (AD) have difficulty reading and spelling irregular words (Cortese, Balota, Sergent-Marshall, \& Buckner, 2003; Strain, Patterson, Graham, \& Hodges, 1998) and generating irregular past tense forms (Cortese et al., 2006; Ullman et al., 1997; although see Lambon Ralph et al., 1995, for an exception to this pattern); and it is known that surface dyslexia can also follow traumatic brain injury (Behrmann \& Bub, 1992; Coltheart, Byng, Masterson, Prior, \& Riddoch, 1983; Marshall \& Newcombe, 1973). The relationship between poor comprehension and "pre-semantic" abilities in stroke aphasia has received even less attention, despite the fact that many stroke aphasic patients have comprehension problems. Furthermore, the few studies that do exist have yielded somewhat contradictory results. At least some of the hallmarks of surface dyslexia - e.g., regularisation errors - have been observed in some comprehension-impaired stroke aphasic patients (e.g., Peach, 2002), but other single-case studies demonstrate that comprehension impairment in stroke aphasia does not always give rise to such a pattern (Gerhand, 2001). At any rate, these patients often have additional phonological deficits that make it difficult to interpret patterns of performance in tasks requiring a spoken response (Peach, 2002). The effect of semantic impairment on other presemantic tasks - for example, past tense generation, spelling, wordor picture-recognition, and delayed picture copying - has scarcely received any attention in stroke aphasia, even though a comparison of tasks that utilise different input and output modalities would aid the interpretation of deficits in patients with additional phonological problems.

\footnotetext{
1 Following the same logic, the results of Patterson et al. (2006) would result from damage to multiple independent subsystems supporting visual word recognition, visual object recognition, visual working memory, spelling, and verb inflection. Although independent deficits to semantics and domain-specific processes could readily account for problems in one task, this account is less viable when all of these domains are considered together: many different domain-specific representations, spanning a very wide range of verbal and non-verbal tasks, would have to be impaired in parallel following circumscribed damage to the anterior temporal cortices. Patterson et al. (2006) further note that this notion of 'associated but unrelated deficits' does not explain why SD patients show regularity effects in all of these tasks.
} 
The current work focuses on two important questions. The first is empirical: Do patients with multimodal semantic impairments from stroke aphasia show the same pattern of impairment on pre-semantic tasks as patients with SD? The second question is more theoretical: If comprehension-impaired stroke aphasics do not show the same patterns of impairment on pre-semantic tasks as SD patients, does this provide evidence against the theory articulated by Patterson et al. (2006), to wit, that pre-semantic processing for low-frequency atypical items is reliant on support from the semantic system? The answer to the latter question depends upon one's hypothesis about the nature of the semantic impairment observed in the two disorders. Below, we describe our working hypothesis about the nature of the semantic impairment in a subset of patients with stroke aphasia who have difficulties comprehending both verbal and non-verbal stimuli (Jefferies, Baker, Doran, \& Lambon Ralph, 2007; Jefferies \& Lambon Ralph, 2006; Jefferies, Patterson, \& Lambon Ralph, 2008). We will discuss some predictions that this hypothesis makes about pre-semantic cognition in this group of patients and then test them in a series of empirical studies. It is important to note that our predictions are specific to patients with multimodal semantic problems: they do not extend to other patterns of comprehension impairment that also occur in stroke aphasia (e.g., difficulty accessing meaning from specific input modalities, such as in pure word deafness; see below). In the general discussion, we will return to the question of whether the observed pattern of results provides evidence for or against the theory developed by Patterson et al. (2006).

\subsection{The nature of multimodal semantic impairment in stroke aphasia}

There are several reasons for believing that multimodal impairment of semantic memory is qualitatively different in SD and stroke aphasia. First, semantic deficits in these two groups are associated with very different areas of brain damage. SD patients have highly circumscribed atrophy of the inferior and lateral aspects of the ATL bilaterally. Hypometabolism in those regions correlates with the severity of the semantic deficit in SD (Mummery et al., 2000; Nestor, Fryer, \& Hodges, 2006). In contrast, semantic impairment in aphasia is associated with damage to temporoparietal and prefrontal regions in the left hemisphere (e.g., Berthier, 2001; Chertkow, Bub, Deaudon, \& Whitehead, 1997; Jefferies \& Lambon Ralph, 2006). The ATL is rarely damaged in patients with stroke aphasia, for two reasons: (i) the ATL often has a double blood supply (the anterior temporal cortical artery of the middle cerebral artery and the anterior temporal branch of the distal posterior cerebral artery: Borden, 2006; Conn, 2003) and (ii) the anterior temporal cortical artery branches below the main trifurcation of the MCA and thus may be less vulnerable to emboli.

Secondly, although these two groups of patients can show similar neuropsychological profiles, they appear to have different underlying reasons for their semantic problems. In a series of recent studies, we have directly compared SD patients with stroke aphasic patients who were selected to show semantic impairment in both verbal and non-verbal tasks (Jefferies et al., 2007; Jefferies \& Lambon Ralph, 2006; Jefferies et al., 2008). Therefore, our aphasic participants had impairment that went beyond language to affect general semantic cognition (as a shorthand, we will refer to this pattern as "semantic aphasia" or SA). Despite the fact that the SD and SA patients obtained comparable scores on the same range of semantic tasks, there were several clear differences in the nature of the semantic deficit: (1) patients with SD were highly sensitive to the frequency/familiarity of the test items, whereas patients with SA showed no such sensitivity.(2) In picturenaming tasks, patients with SD produced many coordinate and superordinate errors but virtually no associative errors, whereas associative errors were common in SA (e.g., squirrel $\rightarrow$ "nuts"; glass $\rightarrow$ "ice"; lorry $\rightarrow$ "diesel"). (3) Though both groups showed impairments across different modalities of testing, patients with SD showed considerable item-wise consistency across different kinds of semantic tasks (e.g., word-picture matching vs. picturematching), whereas patients with SA showed little item-wise consistency on tests requiring different types of semantic judgement. (4) In picture naming, patients with SD showed relatively little benefit from progressive phonological cues, whereas patients with SA showed very substantial increases in naming accuracy following cueing.

From these data, Jefferies and colleagues hypothesized that, whereas SD produces a degradation of amodal semantic representations formed in the ATL (Rogers, Lambon Ralph, Garrard, et al., 2004), semantic impairment in SA results from an inability to appropriately regulate activation within the semantic system, perhaps as the result of damage to fronto-parietal executive/control systems. Such control processes might be required, for instance, to focus on aspects of knowledge that are relevant for a given task or context, to identify semantic relationships that are not prepotent within the semantic system and to select among multiple competing responses (Noonan et al., in press; Saffran, 2000). This hypothesis also appears to be consistent with current theories about the role of prefrontal and parietal regions in semantic cognition, which suggest that these areas are involved in selection and/or controlled retrieval of information from the semantic system (Gold \& Buckner, 2002; Noppeney, Phillips, \& Price, 2004; ThompsonSchill, D’Esposito, Aguirre, \& Farah, 1997; Wagner, Pare-Blagoev, Clark, \& Poldrack, 2001). In line with this view, the semantic difficulties of the SA patients were concomitant with executive impairment, whereas non-verbal reasoning was largely intact in the SD group (see also Baldo et al., 2005; Wiener, Connor, \& Obler, 2004).

This hypothesis about the nature of the semantic impairment in SA vs. SD - namely that semantic representations are degraded in SD, whereas semantic abilities are deregulated in SA - anticipates that "pre-semantic" abilities will not be similarly affected in the two disorders. Specifically, we made three predictions. (1) The deregulation of semantic memory seen in SA should produce less disruption of pre-semantic tasks compared with the semantic degradation that occurs in SD. (2)The SA group should show a lesser degree of sensitivity to regularity in the different pre-semantic tasks. (3) The SA group should produce different kinds of errors in the various tasks, rather than a preponderance of regularisations and LARC errors.

Our reasoning for these predictions was as follows. In SD, poor performance in pre-semantic tasks arises because processing of lower frequency irregular items depends, in the healthy brain, upon robust activation of associated semantic representations in the ATL. Higher frequency items, and items whose structure is generally consistent with the domain (i.e., "regular" items), depend less on this support. Hence, when the ATL becomes severely atrophied in SD, the processing of lower frequency irregular items suffers to a greater extent than the processing of more frequent or more regular items. In SA, the key point is that although control of activation within the semantic system may be disrupted, items in pre-semantic tasks should continue to activate the associated semantic representations in the undamaged ATL. Although the flow of activation through the network may be somewhat unconstrained in SA, input from anterior temporal regions to other parts of the network is not grossly reduced, as it is in SD. Consequently, lower frequency and irregular surface forms continue to receive strong input from the ATL semantic system. Deficits may still arise for several reasons - for instance, from impairment in the ability to discern task-appropriate semantic relationships, to select the correct 
response from among a set of competing alternatives, or to settle upon precisely the correct semantic representation - but these deficits should be both milder in general (prediction 1) and less likely to show the frequency by regularity interaction characteristic of SD (prediction 2). Furthermore, an impairment in regulating activation within the semantic system should lead to more arbitrary and/or associative errors, since inappropriate responses cannot be inhibited (prediction 3).

To test these predictions, we examined the performance of the SA patients reported by Jefferies and Lambon Ralph (2006) on seven "pre-semantic" tasks used previously with SD patients by Patterson et al. (2006) and Rogers, Patterson, and Graham (2007). We directly compared SD and SA patients to establish if they showed differences in the magnitude of regularity/typicality effects in reading, past tense generation, spelling to dictation, lexical decision, object decision, colour decision and delayed picture copying. Semantic degradation in SD impairs all of these tasks in a similar fashion, indicating that conceptual knowledge makes an important contribution to the processing of items that are atypical in each domain (Patterson et al., 2006). We investigated whether this pattern extends to SA patients whose semantic deficits, we suggest, result from a loss of semantic control and not degradation of semantic knowledge per se. If SA patients fail to show an effect of multimodal semantic impairment across these verbal and nonverbal "pre-semantic" tasks, we can conclude that the nature of the semantic impairment and location of brain damage are important mediators in this relationship. This changes the interpretation of SA cases who fail to show predicted deficits such as surface dyslexia. If our hypotheses are correct, then the existence of such patients does not provide evidence for the independence of semantic memory and "pre-semantic" cognition.

\section{Method}

\subsection{Participants}

Thirteen aphasic stroke patients were recruited from stroke clubs and speech and language therapy services in Manchester, York and Harrogate, UK. Every case had chronic impairment from a CVA at least a year previously. The patients were recruited in order to investigate the nature of multimodal semantic impairment following stroke (Jefferies \& Lambon Ralph, 2006). Like SD patients, they failed a range of verbal and non-verbal semantic tasks tapping the comprehension of words, pictures and environmental sounds. They had a variety of aphasia syndromes: seven patients had transcortical sensory aphasia (i.e., poor comprehension in the context of fluent speech and preserved repetition, superficially resembling the pattern seen in SD) and six patients had additional language deficits. Table 1 shows biographi$\mathrm{cal} /$ neuroimaging details and aphasia classifications based on the Boston Diagnostic Aphasia Examination (BDAE; Goodglass, 1983) and some additional repetition tests from the PALPA battery (Kay, Lesser, \& Coltheart, 1992). Table 2 provides additional neuropsychological test scores.

The additional deficits in the SA patient group require some comment in the context of this study. SD is highly unusual in that it produces a selective impairment of semantic memory. All other conditions that affect semantic cognition (e.g.. herpes simplex encephalitis, $\mathrm{AD}, \mathrm{SA}$ ) co-occur with other deficits that vary from individual to individual (e.g., in visual processing, phonology, etc.). Inevitably such additional impairments will contribute to deficits on pre-semantic tasks when these tasks also draw upon the impaired domain-therefore a pragmatic approach is required to answer the core study question. We achieved this in several ways. First, we examined a wide range of pre-semantic tasks that involved different input modalities (coloured pictures, line drawings, written words, spoken words) and output modalities (speech, drawing, writing, pointing). Consequently, for each individual patient, only a subset of these tasks will show an effect of additional deficits. Secondly, in our direct comparisons of SA and SD, the SA group were screened for extra-semantic deficits that might influence performance on each of the pre-semantic tasks. We only included individual SA cases who performed well on these screening assessments.

The SA cases were directly compared with two sets of previously published data from patients with SD to establish whether a range of pre-semantic tasks showed an interaction between patient group and regularity/typicality: (1) six "pre-semantic" tasks were examined in fourteen SD patients by Patterson et al. (2006). (2) A separate group of ten SD patients were tested on an additional colour decision task by Rogers et al. (2007). Basic biographical and neuropsychological details are provided for these two SD groups in Table 3. Additional details are also provided in the original papers.

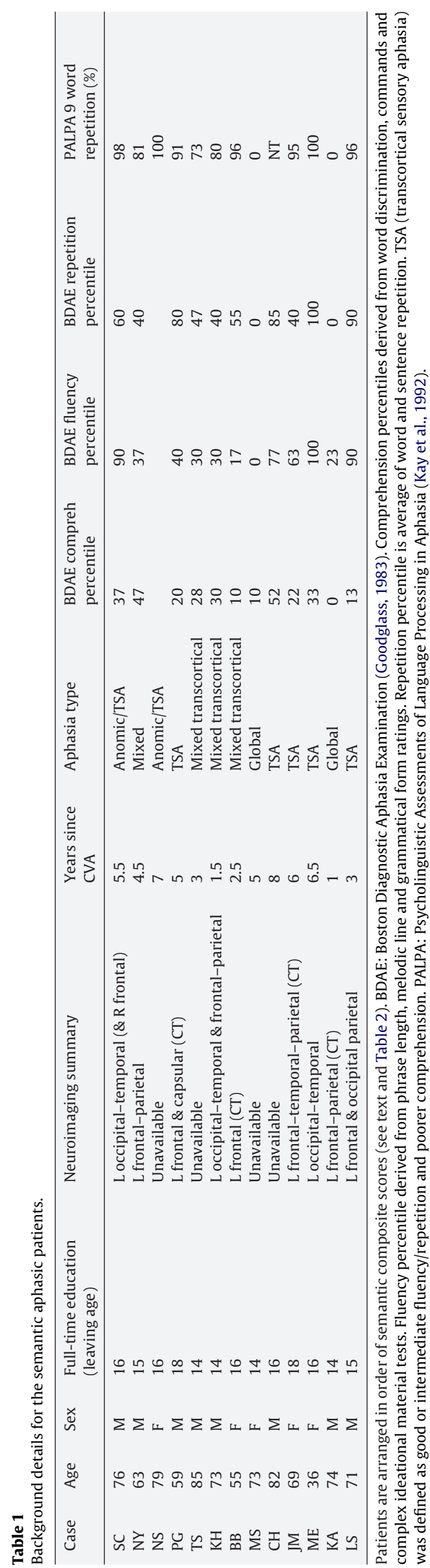


Table 2

Neuropsychological assessment for the SA cases.

\begin{tabular}{|c|c|c|c|c|c|c|c|c|c|c|c|c|c|c|c|c|}
\hline Task & Max & Normal cut-off & SC & NY & NS & PG & TS & $\mathrm{KH}$ & BB & MS & $\mathrm{CH}$ & JM & ME & KA & LS & SA average \\
\hline Composite semantic score & & & 1.4 & 1.2 & 1.0 & 0.6 & 0.6 & 0.2 & 0.2 & -0.2 & -0.3 & -0.4 & -1.2 & -1.2 & -1.8 & \\
\hline Picture PPT & 52 & 48 & 50 & $47^{*}$ & $47^{*}$ & $42^{*}$ & $42^{*}$ & $41^{*}$ & $41^{*}$ & $41^{*}$ & $36^{*}$ & $35^{*}$ & $29^{*}$ & $44^{*}$ & $31^{*}$ & 40.5 \\
\hline Word PPT & 52 & 49 & 51 & $42^{*}$ & NT & $43^{*}$ & NT & $39 *$ & $35^{*}$ & $34^{*}$ & NT & $44^{*}$ & $39 *$ & $44^{*}$ & $39 *$ & 41.0 \\
\hline Picture naming & 64 & 59 & $28^{*}$ & $51^{*}$ & $56^{*}$ & $44^{*}$ & $55^{*}$ & $29^{*}$ & $9 *$ & $0^{*}$ & NT & $30^{*}$ & $4^{*}$ & $0^{*}$ & $5^{*}$ & 25.9 \\
\hline Word-picture matching & 64 & 62 & $59^{*}$ & $60^{*}$ & $57^{*}$ & $58^{*}$ & $58^{*}$ & $54^{*}$ & $54^{*}$ & $46^{*}$ & $53^{*}$ & $53^{*}$ & $50^{*}$ & $26^{*}$ & $37^{*}$ & 51.2 \\
\hline Synonym judgement & 96 & 91 & $71^{*}$ & $69^{*}$ & $81^{*}$ & $69^{*}$ & $69^{*}$ & $61^{*}$ & $61^{*}$ & $51^{*}$ & $81^{*}$ & $69^{*}$ & $81^{*}$ & $60^{*}$ & $48^{*}$ & \\
\hline Letter fluency & - & 22 & $24^{*}$ & $5^{*}$ & NT & $2^{*}$ & $1^{*}$ & $0^{*}$ & $0^{*}$ & $0^{*}$ & NT & $1^{*}$ & $14^{*}$ & $0^{*}$ & $8^{*}$ & \\
\hline Category fluency & - & 62 & $17^{*}$ & $25^{*}$ & NT & $4^{*}$ & $14^{*}$ & $18^{*}$ & $13^{*}$ & $0^{*}$ & NT & $17^{*}$ & $25^{*}$ & NT & $11^{*}$ & \\
\hline VOSP dot counting & 10 & 8 & 10 & 10 & 8 & $5^{*}$ & 10 & 10 & 10 & 10 & NT & 10 & $3^{*}$ & $0^{*}$ & $6^{*}$ & \\
\hline VOSP position discrimination & 20 & 18 & $17^{*}$ & 20 & $16^{*}$ & 20 & 19 & 18 & 18 & 19 & NT & 19 & $15^{*}$ & $14^{*}$ & $16^{*}$ & \\
\hline VOSP number location & 10 & 7 & 10 & 10 & 9 & 9 & $6^{*}$ & 9 & 8 & NT & NT & $5^{*}$ & $2^{*}$ & $6^{*}$ & 8 & \\
\hline VOSP cube analysis & 10 & 6 & 9 & $5^{*}$ & $5^{*}$ & 10 & $5^{*}$ & $3^{*}$ & $2^{*}$ & 8 & NT & $3^{*}$ & $4^{*}$ & NT & $4^{*}$ & \\
\hline Raven's coloured matrices & 36 & $14^{\dagger}$ & 22 & 26 & $12^{*}$ & 23 & 16 & $12^{*}$ & 24 & $12^{*}$ & 25 & $14^{*}$ & $13^{*}$ & $12^{*}$ & 16 & \\
\hline Brixton spatial anticipation (correct) & 54 & 28 & $25^{*}$ & 34 & $15^{*}$ & $26^{*}$ & $18^{*}$ & $7^{*}$ & $23^{*}$ & $16^{*}$ & NT & NT & $11^{*}$ & $6^{*}$ & $14^{*}$ & \\
\hline TEA: counting without distraction & 7 & 6 & 7 & $3^{*}$ & NT & $3^{*}$ & $4^{*}$ & 6 & $4^{*}$ & NT & NT & $3^{*}$ & 7 & NT & $3^{*}$ & \\
\hline TEA: counting with distraction & 10 & 3 & $1^{*}$ & $2^{*}$ & NT & $0^{*}$ & $1^{*}$ & 3 & $0^{*}$ & NT & NT & $0^{*}$ & 9 & NT & $2^{*}$ & \\
\hline Digit span: forwards & - & 5 & 6 & $3^{*}$ & 4 & 6 & $3^{*}$ & $4^{*}$ & 5 & $0^{*}$ & 7 & 3* & 6 & $0^{*}$ & $4^{*}$ & \\
\hline Digit span: backwards & - & 2 & 2 & 2 & 2 & 2 & 2 & 2 & $0^{*}$ & NT & NT & 2 & 3 & NT & $1^{*}$ & \\
\hline
\end{tabular}

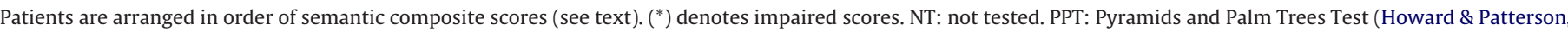

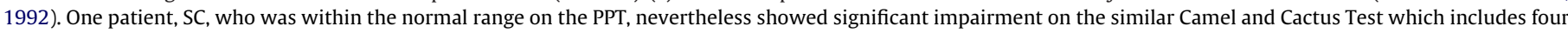
response options (Bozeat et al., 2000). For non-standardised assessments, normal cut-off $=2$ SD below control mean. ${ }^{\dagger}$ Cut-off for Raven's corresponds to 5 th percentile.

\subsection{Assessments}

\subsubsection{General neuropsychology}

Table 2 provides background scores for the SA patients on a range of semantic and non-semantic tasks, allowing comparison with the neuropsychological profile in SD. In addition to the BDAE and repetition tests from the PALPA (see above), the patients were examined on the Visual Object and Space Perception battery (Warrington \& James, 1991), forwards and backwards digit span (Wechsler, 1987) and two attentional/executive tests-the Coloured Progressive Matrices test of nonverbal reasoning (Raven, 1962) and the Elevator Counting subtests with and without distraction from the Test of Everyday Attention (Robertson, Ward, Ridgeway, \& Nimmo-Smith, 1994).

Semantic processing was assessed with the following tests: the Pyramids and Palm Trees Test (PPT), in which subjects decide which of two items is more associated with a target (e.g., pyramid with pine tree or palm tree) for pictures and for words (presented simultaneously as spoken and written forms) in two separate versions (Howard \& Patterson, 1992); word-picture matching (with ten choices from the same semantic category); synonym judgement for words varying in frequency and imageability with three choices per trial (the items were presented as both written and spoken words; see Jefferies et al., 2009); naming a set of 64 black and white line drawings from Snodgrass and Vanderwart (1980) set; category fluency for six categories (animals, birds, fruit, household items, tools and vehicles) and verbal fluency for three letters (F, A, S). In both fluency tests, participants produced as many exemplars as possible within $1 \mathrm{~min}$.

An overall measure of semantic impairment was derived using factor analysis using semantic assessments that had been completed by all patients and that did not involve spoken output (picture PPT; word-picture matching). The first and only factor in the solution accounted for $53.8 \%$ of the total variance. Scores on this factor were computed for each individual patient and used as a composite measure of semantic impairment. The patients are ordered by this variable in the data tables below.

Table 3

Background details for SD cohorts.

\begin{tabular}{|c|c|c|c|c|c|c|c|c|c|}
\hline & Case & Age & Sex & MMSE & Rey & $\begin{array}{l}\text { Picture name } \\
\text { (out of } 64 \text { ) }\end{array}$ & $\begin{array}{l}\text { Word-picture } \\
\text { match (out of } 64 \text { ) }\end{array}$ & $\begin{array}{l}\text { Picture PPT } \\
\text { (out of } 52 \text { ) }\end{array}$ & $\begin{array}{l}\text { Composite } \\
\text { semantic }\end{array}$ \\
\hline \multirow[t]{14}{*}{ Cohort 1} & AN & 65 & M & 27 & 36 & 41 & 62 & 48 & 1.54 \\
\hline & $\mathrm{JP}$ & 66 & M & 26 & 36 & 49 & 63 & 47 & 1.49 \\
\hline & JTh & 55 & $F$ & 25 & 31 & 43 & 55 & 49 & 1.37 \\
\hline & SJ & 60 & $\mathrm{~F}$ & 19 & 34 & 11 & 51 & 45 & 0.89 \\
\hline & DV & 65 & M & 23 & 36 & 15 & 49 & 41 & 0.48 \\
\hline & NS & 69 & $F$ & 25 & 36 & 8 & 42 & 39 & 0.06 \\
\hline & LS2 & 61 & M & 24 & 29 & 34 & 60 & 29 & -0.14 \\
\hline & EK & 60 & $\mathrm{~F}$ & 27 & 35 & 17 & 43 & 33 & -0.41 \\
\hline & BS & 68 & M & 25 & 33 & 29 & 40 & 33 & -0.52 \\
\hline & JTw & 66 & M & 25 & 31 & 5 & 34 & 35 & -0.57 \\
\hline & WM & 55 & $\mathrm{~F}$ & 21 & 34 & 6 & 21 & 38 & -0.78 \\
\hline & KI & 65 & M & 23 & 34 & 15 & 36 & 31 & -0.83 \\
\hline & ATe & 62 & M & 20 & 36 & 3 & 29 & 31 & -1.08 \\
\hline & JG & 71 & $\mathrm{~F}$ & 19 & 34 & 2 & 8 & 35 & -1.50 \\
\hline \multirow[t]{10}{*}{ Cohort 2} & AN & 65 & M & 28 & 36 & 53 & 63 & & \\
\hline & $\mathrm{JP}$ & 66 & M & 26 & 36 & 49 & 63 & & \\
\hline & LS & 61 & M & 25 & 36 & 36 & 62 & & \\
\hline & $\mathrm{JT}$ & 55 & $\mathrm{~F}$ & 25 & 31 & 43 & 55 & & \\
\hline & WM & 54 & $\mathrm{~F}$ & 24 & 36 & 14 & 52 & & \\
\hline & ATh & 61 & M & 13 & 34 & 13 & 45 & & \\
\hline & NS & 69 & $\mathrm{~F}$ & 25 & 36 & 8 & 42 & & \\
\hline & $\mathrm{JCh}$ & 59 & M & 12 & 25 & 14 & 37 & & \\
\hline & ATe & 67 & M & 24 & 36 & 5 & 29 & & \\
\hline & JG & 71 & $\mathrm{~F}$ & 8 & 35 & NT & 8 & & \\
\hline
\end{tabular}

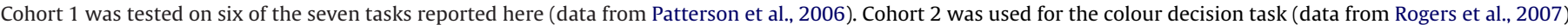

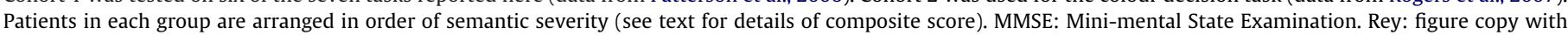
stimulus present. PPT: Pyramids and Palm Trees Test (Howard and Patterson, 1992). 
Table 4

Inclusion and exclusion of SA patients for each task.

\begin{tabular}{|c|c|c|c|c|c|c|c|c|c|c|c|c|c|c|}
\hline Task & Screening measure & SC & NY & NS & PG & TS & $\mathrm{KH}$ & BB & MS & $\mathrm{CH}$ & JM & $\mathrm{ME}$ & $\mathrm{KA}$ & LS \\
\hline Lexical decision & & $\mathrm{Y}$ & $\mathrm{Y}$ & $\mathrm{Y}$ & $\mathrm{Y}$ & $\mathrm{Y}$ & $\mathrm{Y}$ & $\mathrm{Y}$ & $\mathrm{Y}$ & $\mathrm{Y}$ & $\mathrm{Y}$ & $\mathrm{Y}$ & $\mathrm{Y}$ & $\mathrm{Y}$ \\
\hline Object decision & & $\mathrm{Y}$ & $\mathrm{Y}$ & $\mathrm{Y}$ & $\mathrm{Y}$ & $\mathrm{Y}$ & $\mathrm{Y}$ & $\mathrm{Y}$ & $\mathrm{Y}$ & $\mathrm{Y}$ & $\mathrm{Y}$ & $\mathrm{Y}$ & $\mathrm{Y}$ & $\mathrm{Y}$ \\
\hline Colour decision & & $\mathrm{Y}$ & $\mathrm{Y}$ & Y & $\mathrm{Y}$ & $\mathrm{Y}$ & $\mathrm{Y}$ & $\mathrm{Y}$ & NT & $\mathrm{Y}$ & NT & Y & Y & $\mathrm{Y}$ \\
\hline Reading & $>75 \%$ regular items from PALPA 35 correct & Y & Y & $\mathrm{Y}$ & Y & Y & $\mathrm{N}$ & $\mathrm{N}$ & $\mathrm{N}$ & $\mathrm{Y}$ & NT & $\mathrm{N}$ & $\mathrm{N}$ & $\mathrm{N}$ \\
\hline Spelling & $4 / 6$ "primer" words from BDAE correct & $\mathrm{Y}$ & Y & $\mathrm{N}$ & $\mathrm{N}$ & $\mathrm{Y}$ & $\mathrm{N}$ & $\mathrm{N}$ & $\mathrm{N}$ & $\mathrm{Y}$ & NT & Y & $\mathrm{N}$ & $\mathrm{N}$ \\
\hline Past tense & Good speech production-e.g., PALPA reading & $\mathrm{Y}$ & Y & $\mathrm{Y}$ & $\mathrm{N}$ & $\mathrm{N}$ & $\mathrm{N}$ & $\mathrm{N}$ & $\mathrm{N}$ & $\mathrm{Y}$ & NT & Y & $\mathrm{N}$ & $\mathrm{N}$ \\
\hline Picture copying & $>75 \%$ features correct in immediate copy & Y & $\mathrm{Y}$ & NT & $\mathrm{Y}$ & NT & NT & $\mathrm{Y}$ & NT & NT & NT & $\mathrm{N}$ & $\mathrm{N}$ & Y \\
\hline
\end{tabular}

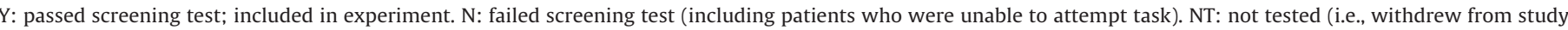
prior to experiment).

\subsubsection{Experimental pre-semantic tasks}

There were four verbal tasks (lexical decision, reading aloud, spelling to dictation, and past tense generation) and three non-verbal tasks (object decision, colou decision, and delayed picture copying). Six of these tasks were used by Patterson et al. (2006) to examine the status of "pre-semantic" tasks in SD. The additional colour decision task was presented to patients with SD by Rogers et al. (2007). This assessment took the same form as the object and lexical decision tasks but there is arguably less controversy about whether this task requires access to semantic information. The tasks requiring active production (reading aloud, spelling to dictation, past tense generation, and delayed picture copying) were only examined in a subset of patients with relatively good output in the required domain. Table 4 details which patients were included for each task.

2.2.2.1. Lexical decision. All thirteen patients completed this two-alternative forced-choice (2AFC) task, first used by Rogers, Lambon Ralph, Hodges, et al. (2004). There were 72 pairs of items, with a word and a nonword in each. The nonword was a pseudohomophone of the word. The words were selected to be high and low frequency. Typicality was manipulated by selecting pairs in which the word was more or less typical than the nonword: for example, node/gnode was presented in the $\mathrm{W}>\mathrm{NW}$ condition, with gnome/nome in the $\mathrm{NW}>\mathrm{W}$ condition, as/n/is more commonly spelled ' $n$ ' than 'gn'. The pairs of items were presented on cards with the words/nonwords on the left/right equally often. Participants were asked to "point to the real word" on each trial.

2.2.2.2. Object decision. All thirteen patients completed this $2 \mathrm{AFC}$ task described by Rogers et al. (Rogers et al., 2003; Rogers, Lambon Ralph, Hodges, et al., 2004). There were 60 trials that consisted of a pair of line drawings; one was a real object and one was a non-real version of the same object. The objects were selected to have high and low rated familiarity/frequency. As in the lexical decision test, typicality was manipulated by selecting pairs in which the real object was more or less typical than the non-real version. For example, in the $\mathrm{R}>\mathrm{NR}$ condition, there was a normal monkey vs. a monkey with large elephant-sized ears, and in the NR $>$ R condition, there was a normal elephant vs. an elephant with monkey-sized ears. Animals tend to have small ears so ear-size is atypical for elephants. As before, the pairs were presented on cards with the typical and less typical items on the left/right equally often. Participants were asked to "point to the real one".

2.2.2.3. Colour decision. Eleven patients performed a $2 \mathrm{AFC}$ task first used by Rogers et al. (2007). Patients were asked to decide which of two pictures was coloured correctly. Again, typicality was manipulated. In the R $>$ NR condition, the correct choice had a colour typical for objects in that category (either brown for animals or green for plants) while the distractor had an atypical colour (for example, a green lettuce was shown alongside a red lettuce). In the NR $>\mathrm{R}$ condition, the target had a colour that was not typical while the distractor was typical of the domain (e.g., a red strawberry was shown alongside a green strawberry). The pairs were presented on cards with the typical and less typical items on the left/right equally often. Target items in the R $>$ NR and NR $>$ R conditions were matched for frequency. There were 15 trials in each condition. There were also 18 filler trials included to increase the range of correct-choice colours. Further methodological details are given in Rogers et al. (2007).

2.2.2.4. Reading aloud. Patients were screened for the ability to read aloud regular words. Specifically, patients who were unable to read at least $75 \%$ of the regular words from PALPA 35 (which includes 60 regular and irregular words; Kay et al., 1992) were excluded from testing on the experimental reading measure. This included five patients with very limited or error-prone spoken output. One further patient (ME) with good spoken output refused to attempt the task, explaining that she could not 'see' the words-perhaps as a consequence of visual impairment following from her occipital-temporal lesion. Six patients were tested on the regularity by frequency items used by Patterson et al. (2006) in their sample of SD patients. There were 168 single-syllable words that crossed word frequency (high vs. low) and typicality (regular vs. irregular spelling-sound correspondences), with 42 items in each set. The words were presented individually in a random order.
2.2.2.5. Spelling to dictation. As a screening task, we asked patients to spell the high frequency "primer" words in the BDAE (Goodglass, 1983). We excluded patients who were unable to spell at least four of the six items correctly (see Table 4). Five patients completed the harder spelling test used by Patterson et al. (2006) with SD patients. There were 36 single-syllable words that crossed frequency (high, medium, and low) with typicality (regular vs. irregular), with six items in each set. The medium and low-frequency words in each of the regularity conditions were combined into a low-frequency set in the analysis, following Patterson et al. (2006) treatment of the SD data. The items were dictated one at a time in a random order. Patients repeated the items before trying to write them down them to ensure that they had been heard correctly.

2.2.2.6. Past tense generation. Patients with limited or error-prone speech production - all of whom failed the screening task for reading aloud - were excluded from consideration in this task, since it required spoken output. Five SA patients were compared with the SD group. One hundred verbs from Patterson et al. (2001) were used. There were 25 items in four conditions that crossed word frequency (high vs. low) and typicality (regular vs. irregular). Participants heard and saw a short sentence in which the target verb was used in its stem/present-tense form (e.g. "Today I eat lunch"), followed by a second sentence in the past tense with a gap where the verb should be (e.g., "Yesterday I ___-_ lunch"). The patients were asked to reproduce the verb from the first sentence but this time in the past tense. Practice trials before the experiment were used to ensure that the patients understood the task.

2.2.2.7. Picture copying. Seven patients were asked to copy 24 line drawings both when the picture was in front of them and after a delay. The immediate copy condition was used a screening measure to identify patients who had problems perceiving the pictures or executing the drawings. We included five patients in the delayed-copy experiment who correctly reproduced $75 \%$ of the drawings' features in their immediate copies (see below for scoring details). In the delayed copying task, SD and SA patients were shown the line drawings and asked to remember them whilst counting aloud up from one with the experimenter for approximately $10 \mathrm{~s}$. After the delay, the patients were asked to try to reproduce the pictures from memory (picture names were not provided at any point). The SD patients tested by Patterson et al. (2006) did not perform the immediate copy task, although both immediate and delayed copy in SD were investigated by Bozeat et al. (2003), and Rogers, Lambon Ralph, Garrard, et al. (2004), with both studies finding essentially normal immediate copy performance in this disorder.

Picture copying was initially scored following the method devised by Bozeat et al. (2003) for SD patient data. A checklist of the features produced by every control subject in delayed copying was produced. Features generated by the patients were counted as correct if they were in this checklist. Omissions occurred when the patients missed out a feature that had been included universally by the controls. Feature intrusion errors occurred when the patients produced a feature that was not in the original drawing or checklist. Each target feature was further classified as shared within domain, shared within category, or distinctive. The first group comprised features that were common to more than half of the items in the same superordinate category (e.g., for animals, features such as eyes, mouths and tails; for artefacts, general properties like having handles or corners). The second level of distinctiveness comprised features that were common to more than half of the items in the same basic category: for example, feathers and wings are common to most birds, but not most animals; wheels and doors are common to cars but not other artefacts. Finally, distinctive features tended not to be present in other semantically related items (e.g., a camel's hump).

As this coding scheme was specifically developed to capture the aspects of drawing performance that breakdown in SD, we devised a supplementary scoring scheme to explore other aspects of the drawings: namely contour violations, vagueness of features and additions of unclassifiable features. To provide a direct comparison with the SA participants, we also analysed picture copying for three further SD patients who had completed both immediate and delayed conditions (one of whom was included in Patterson et al.'s, 2006 study). These additional aspects of drawing performance were initially coded by a single experimenter. The SA and SD patients included unclassifiable intrusions at a similar rate and the features in their delayed 

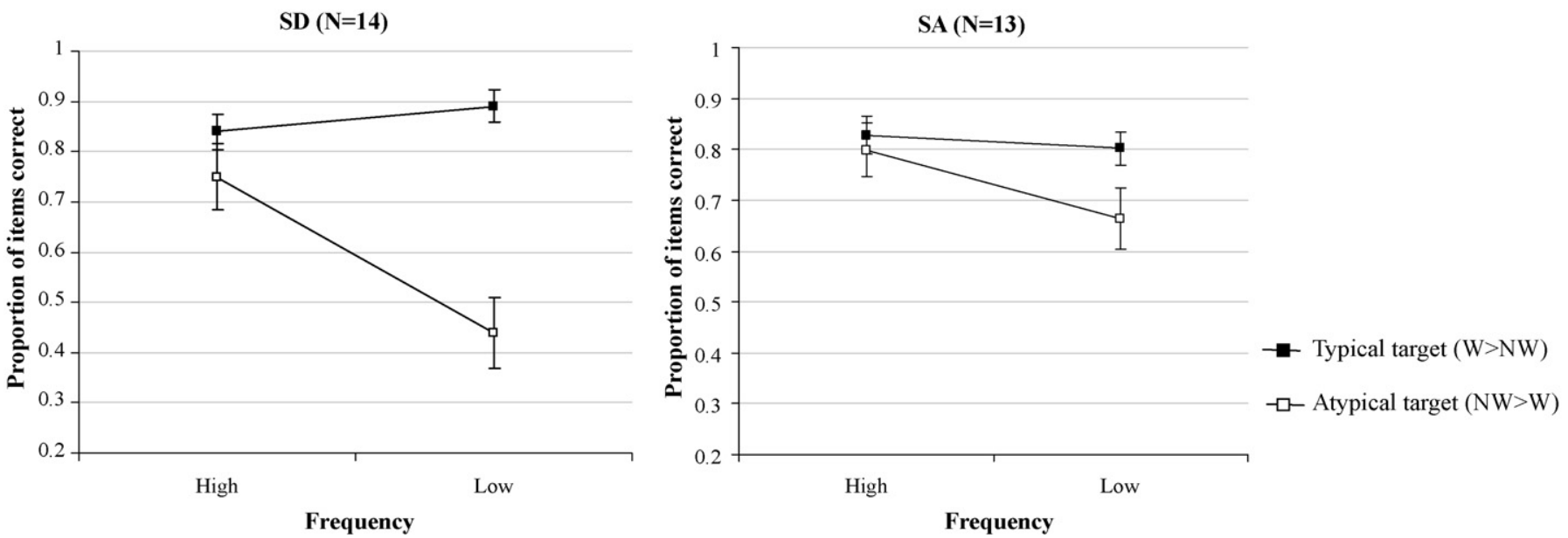

Fig. 1. Effect of frequency and typicality on lexical decision.

Table 5

Summary of individual SA patient performance on the 2AFC tasks.

\begin{tabular}{|c|c|c|c|c|c|c|c|c|c|c|c|c|c|c|}
\hline Task & Condition & SC & NY & NS & PG & TS & $\mathrm{KH}$ & BB & MS & $\mathrm{CH}$ & JM & $\mathrm{ME}$ & $\mathrm{KA}$ & LS \\
\hline Lexical decision & $\mathrm{W}>\mathrm{NW}$ & .75 & .83 & .94 & .97 & .67 & .69 & .72 & .81 & 1 & .86 & .72 & .83 & .81 \\
\hline Lexical decision & $\mathrm{NW}>\mathrm{W}$ & .64 & .72 & .89 & .89 & .72 & .22 & .69 & .69 & .89 & 1 & .78 & .72 & .64 \\
\hline Object decision & $\mathrm{R}>\mathrm{NR}$ & 1 & .97 & .93 & .97 & .90 & 1 & 1 & 1 & .77 & 1 & .90 & .87 & .93 \\
\hline Object decision & $N R>R$ & .9 & .87 & .83 & 1 & .93 & .97 & .90 & .93 & .70 & .97 & .90 & .70 & .97 \\
\hline Colour decision & $\mathrm{R}>\mathrm{NR}$ & .80 & .73 & .80 & .73 & .87 & .87 & 1 & NT & .73 & NT & .87 & .73 & .80 \\
\hline Colour decision & $N R>R$ & .73 & .87 & .73 & .93 & .80 & .60 & .87 & NT & .53 & NT & .87 & .53 & .87 \\
\hline
\end{tabular}

The table shows the proportion of trials correct.

drawings were equally vague. Therefore, we focused our formal analysis on contour violations. A second experimenter generated ratings for this property and the interrater reliability was calculated (Kappa $=.77, p<.0001$ ). We used a three-point scale where a score of 1 denoted no contour violations, 2 denoted some breakdown of the object's outline and 3 denoted serious contour violations such as the omission of major parts. We used an average of the scores generated by the two experimenters when there was a discrepancy in their ratings.

\section{Results}

The degree of semantic impairment, as assessed by background semantic tests, was equivalent for the SA cases and SD patients (see Table 2). There were no significant differences in word-picture matching, $t(25)=1.7, p>.1$, or picture PPT, $t(25)<1$, between the SA group and the SD patients examined by Patterson et al. (2006). Similarly, the SD patients who completed the colour decision task
(Rogers et al., 2007) performed at a comparable level to the SA patients on word-picture matching, $t(17)<1$.

\subsection{Summary of previous $S D$ results}

The fourteen SD patients studied by Patterson et al. (2006) showed significant main effects of typicality/regularity and frequency and an interaction between these variables for lexical decision, object decision, reading aloud, spelling to dictation and past tense generation. These patients also showed a typicality effect in delayed picture copying (frequency was not manipulated in this task). In the colour decision task examined by Rogers et al. (2007), the SD patients with more severe semantic deficits (as measured by word-picture matching) performed at floor. Consequently, the effect of typicality did not reach significance in the SD group as a

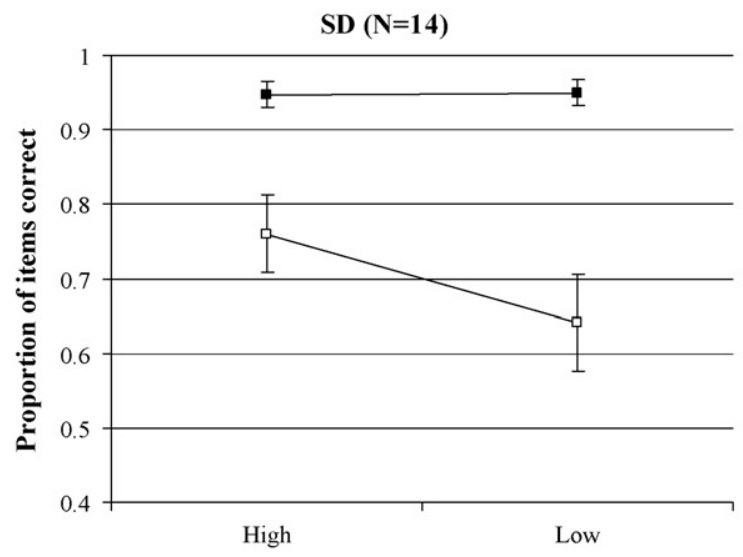

Frequency

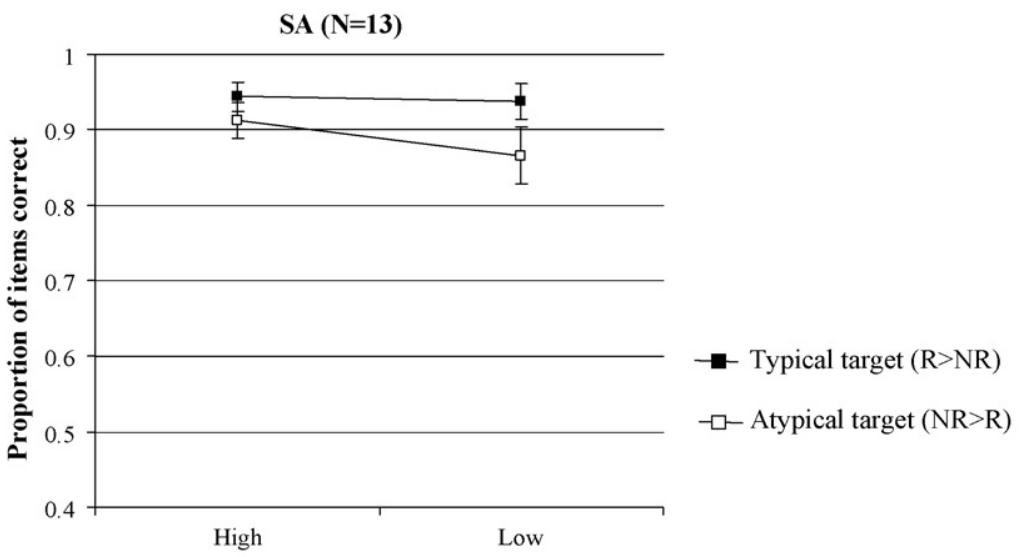

Frequency

Fig. 2. Effect of frequency and typicality on object decision. 
whole $(N=10)$ but the patients with milder SD $(N=5)$ showed a highly significant advantage for the typical items. Frequency was not manipulated in this test.

\subsection{SD vs. SA group comparisons}

\subsubsection{Lexical decision}

A $2 \times 2 \times 2$ mixed factors ANOVA was used to examine the effects of typicality (the difference between $\mathrm{W}>\mathrm{NW}$ and $\mathrm{NW}>\mathrm{W}$ trials), target word frequency and patient group (SD: $N=14$; SA: $N=13$ ). The critical typicality by patient group interaction was significant: the SA patients showed smaller effects of typicality than the SD patients (despite showing a comparable degree of semantic impairment), $F(1,25)=10.7, p=.003$. Although there was no overall difference between the two groups in the effect of frequency $(F(1,25)=1.8, n . s$. $)$, the SD patients showed a larger frequency by typicality interaction than the SA cases, $F(1,25)=12.7, p=.002$. This group comparison is shown in Fig. 1. The SA group as a whole showed an effect of typicality in lexical decision that approached significance, $F(1,12)=4.3, p=.06$, but only a single patient, $\mathrm{KH}$, showed a significant advantage for $\mathrm{W}>\mathrm{NW}$ over $\mathrm{NW}>\mathrm{W}$ trials, Fisher's exact test, one-tailed $p<.0001$ (scores for individual SA patients are provided in Table 5). The SA group did show a significant effect of item frequency, $F(1,12)=9.4, p=.01$ and a regularity by frequency interaction that approached significance, $F(1,12)=3.6$, $p=.08$.

\subsection{Object decision}

In the object decision task, both patient groups were at or near ceiling for the typical target $(R>N R)$ trials. In contrast, the more severe $\mathrm{SD}$ patients were markedly impaired for the atypical target $(\mathrm{NR}>\mathrm{R})$ trials, while performance in the SA group remained relatively good (although 9/13 patients were below the ceiling-level performance reported for healthy older controls by Rogers, Lambon Ralph, Hodges, et al. (2004); individual SA patient performance is summarised in Table 5). A $2 \times 2 \times 2$ mixed factors ANOVA was used to examine the effects of typicality (the difference between $\mathrm{R}>\mathrm{NR}$ and NR $>$ R trials), item frequency and patient group (SD: $N=14$; $\mathrm{SA}: N=13$ ). The typicality by patient group interaction was significant, with the SA patients showing less sensitivity to typicality than the SD cases, $F(1,25)=12.2, p=.002$. However, there was no frequency by group interaction, $F(1,25)<1$. The three-way interaction also failed to reach significance, $F(1,25)=2.8, p=.1$. The SA group as a whole did show a significant typicality effect, $F(1,12)=8.8$, $p=.01$ although this difference did not reach significance for any of the patients individually. The group comparison is shown in Fig. 2.

\subsection{Colour decision}

A $2 \times 2$ mixed factors ANOVA was used to examine the effect of typicality (the difference between $\mathrm{R}>\mathrm{NR}$ and NR $>\mathrm{R}$ trials) and patient group (SD: $N=10$; SA: $N=11$; item frequency was not manipulated). There was no group by typicality interaction when all of the patients were included, presumably because only SD patients with mild semantic impairment showed a significant typicality effect (with more impaired patients performing at chance levels). However, when the five SD patients with the mildest semantic deficits (according to performance on word-picture matching) were compared with the six least-impaired SA patients, a typicality by patient group interaction emerged, $F(1,9)=10.8, p=.009$, even though these subgroups of patients were well-matched in terms of their background semantic scores (comparison for word-picture matching, $t(9)<1$ ). The SA group did not show typicality effects in this task, regardless of whether the analysis included the whole

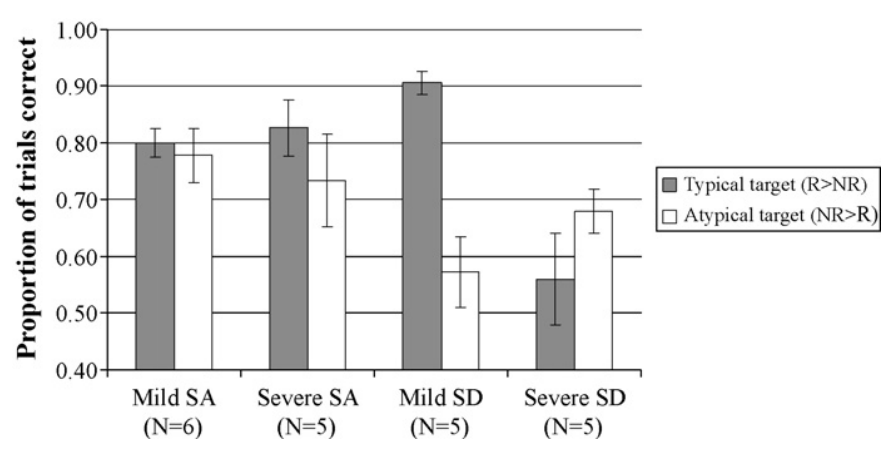

Fig. 3. Effect of typicality on colour decision.

group or only the least semantically impaired patients. This group comparison is shown in Fig. 3. Individual SA patient data are provided in Table 5 .

\subsection{Reading aloud}

Table 6 shows performance on PALPA 35, used here as a screening assessment. Of the nine SA patients who completed this task, six had largely accurate reading of both regular and irregular words, one showed a regularity effect (SC: Fisher's exact one-tailed $p=.02$ ) and two had very poor reading of both regular and irregular items (KH and LS). The majority of the SA patients' errors were visual/phonological errors that were not plausible pronunciations of the target (e.g., pump $\rightarrow$ "kump"). Most of the SA patients also made a few errors that were plausible mispronunciations, i.e., the letter components were given speech sounds that they take in other words. Some of these errors were regularisations (e.g., come $\rightarrow$ "comb") and others were LARC errors ("legitimate alternative reading of components' such as routine $\rightarrow$ "roe-tine"; here the 'ou' segment has been read in line with its pronunciation in 'soul'). In every SA patient, implausible pronunciations greatly outnumbered plausible errors. In contrast, Patterson et al. (2006) found that the overwhelming majority of SD patients' errors in reading aloud were regularisations or LARC errors. The SA patients also produced some additional types of errors: there were small numbers of semantic (wolf $\rightarrow$ "monkey") and/or derivational errors (e.g., iron $\rightarrow$ "ironing"), SC and $\mathrm{KH}$ produced letter naming responses (gauge $\rightarrow G, A, U, G$ ), omissions were common for $\mathrm{KH}$, and LS and $\mathrm{KH}$ made frequent perseverations and unrelated responses.

Six SA patients who performed well in the screening phase also completed the task used by Patterson et al. (2006), allowing a direct comparison with SD. Three SA patients showed a moderate yet significant advantage for reading regular over irregular words (SC: $75 \%$

Table 6

Reading accuracy and errors for PALPA 35 set.

\begin{tabular}{lccccccccl}
\hline & SC & NY & NS & PG & TS & KH & CH & JM & LS \\
\hline Correct: regular & .80 & .90 & .97 & .93 & .87 & .07 & .93 & .97 & 0 \\
Correct: irregular & .50 & .93 & 1 & .93 & .83 & .07 & .90 & .87 & 0 \\
Correct: total & .65 & .92 & .98 & .93 & .85 & .07 & .92 & .92 & 0 \\
Plausible (regularisation + LARC) & .10 & .02 & 0 & .03 & .02 & .02 & 0 & .02 & 0 \\
Non-plausible phonological & .15 & .07 & .02 & .02 & .08 & .12 & .08 & .07 & 0 \\
Semantic + derivational & .02 & 0 & 0 & .02 & .02 & .08 & 0 & 0 & 0 \\
Unrelated & .02 & 0 & 0 & 0 & .02 & .12 & 0 & 0 & .18 \\
Letter sounding/naming & .07 & 0 & 0 & 0 & 0 & .12 & 0 & 0 & 0 \\
Omission & 0 & 0 & 0 & 0 & .02 & .38 & 0 & 0 & 0 \\
Perseveration & 0 & 0 & 0 & 0 & 0 & .10 & 0 & 0 & .82 \\
\hline
\end{tabular}

Data are expressed as a proportion of items presented. Self-corrections were counted as correct (but only one patient, SC, made significant numbers of these). Patients are arranged in order of semantic composite scores (see text). 

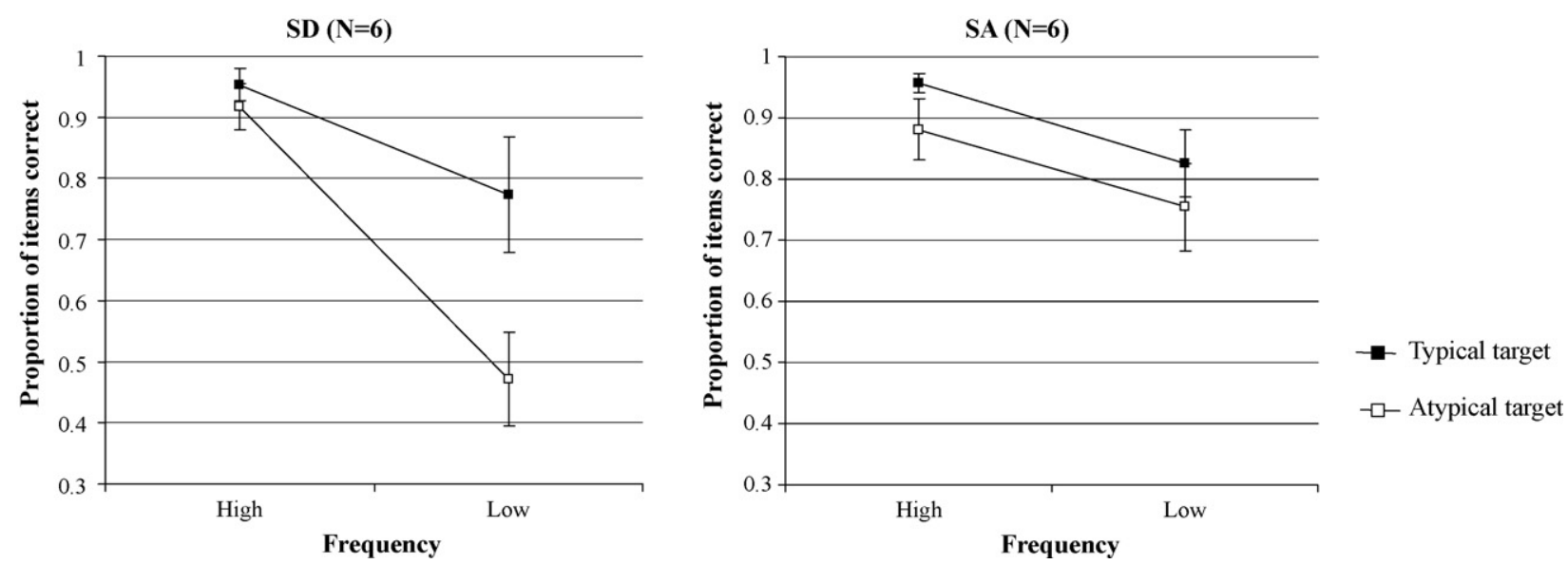

Fig. 4. Effect of frequency and regularity on reading aloud.

vs. $61 \%$ correct, Fisher's exact one-sided $p=.03$; NY: $89 \%$ vs. $76 \%$ correct, $p=.02$; TS: $86 \%$ vs. $73 \%$ correct, $p=.03$ ). The performance of the remaining three SA patients on both regular and irregular items was close to ceiling-in contrast, SD patients with a comparable degree of semantic impairment produced substantial numbers of errors on the low-frequency irregular items. A $2 \times 2 \times 2$ mixed factors ANOVA was used to examine the effects of regularity, item frequency and patient group (see Fig. 4). This analysis only included the six SD patients with the mildest semantic deficits who obtained similar semantic test scores to the SA group (comparison of word-picture matching and picture PPT scores for two subgroups: $t(10)<1.2$ ). Importantly, there was a regularity by patient group interaction: the effect of regularity was significantly stronger in the SD than the SA group, $F(1,10)=8.6, p=.02$. The SD patients also showed a greater influence of frequency on reading accuracy than the SA cases, $F(1,10)=6.8, p=.03$; moreover, the three-way interaction between regularity, word frequency and group was significant, $F(1,10)=11.7, p=.007$. When considered alone, the SA group did show significant effects of both regularity, $F(1,5)=6.8, p=.05$, and frequency, $F(1,5)=10.8, p=.02$.

\subsection{Spelling to dictation}

Of the five SA patients who completed the spelling to dictation task used by Patterson et al. (2006), none showed a significant difference in accuracy for regular and irregular items. Overall accuracy was $17 \%$ for SC, $39 \%$ for NY, $25 \%$ for TS, $89 \%$ for $\mathrm{CH}$ and $47 \%$ for ME. These scores were within the range seen in the SD group. A
$2 \times 2 \times 2$ mixed factors ANOVA was used to examine the effects of regularity, item frequency and patient group (see Fig. 5). This analysis included the six SD patients with the mildest semantic deficits who showed the same degree of semantic impairment as the SA cases (comparison of word-picture matching and picture PPT for two subgroups: $t(9)<1$ ). The interaction between regularity and patient group was again highly significant-the SD patients showed a significantly larger regularity effect than the SA cases, $F(1,9)=14.1, p=.005$. No other interactions reached significance. The SA group alone did show an effect of frequency, $F(1,4)=12.3$, $p=.03$, but no influence of regularity.

NY, SC, CH and TS made primarily non-LARC errors in spelling to dictation: $67-100 \%$ of their incorrect responses were of this type (i.e., the sound segments received non-plausible spellings that are not observed in other words). These errors included letter substitutions (e.g., yeast $\rightarrow$ neast), deletions (e.g., snatch $\rightarrow$ sntch), additions (e.g., couch $\rightarrow$ cousqud) and migrations (e.g., couch $\rightarrow$ choc). NY and TS also made omission errors. In contrast, 74\% of ME's errors were LARC responses. This pattern is in line with the SD patients studied by Patterson et al. (2006).

\subsection{Past tense generation}

Two of the six SA cases who completed the past tense generation task showed a significant regularity effect (both NY and SC: $64 \%$ vs. $44 \%$ correct for regular and irregular verbs respectively; Fisher's exact one-sided $p<.04)$. NY also showed significantly better performance for high than low-frequency verbs (NY $=64 \%$ vs.
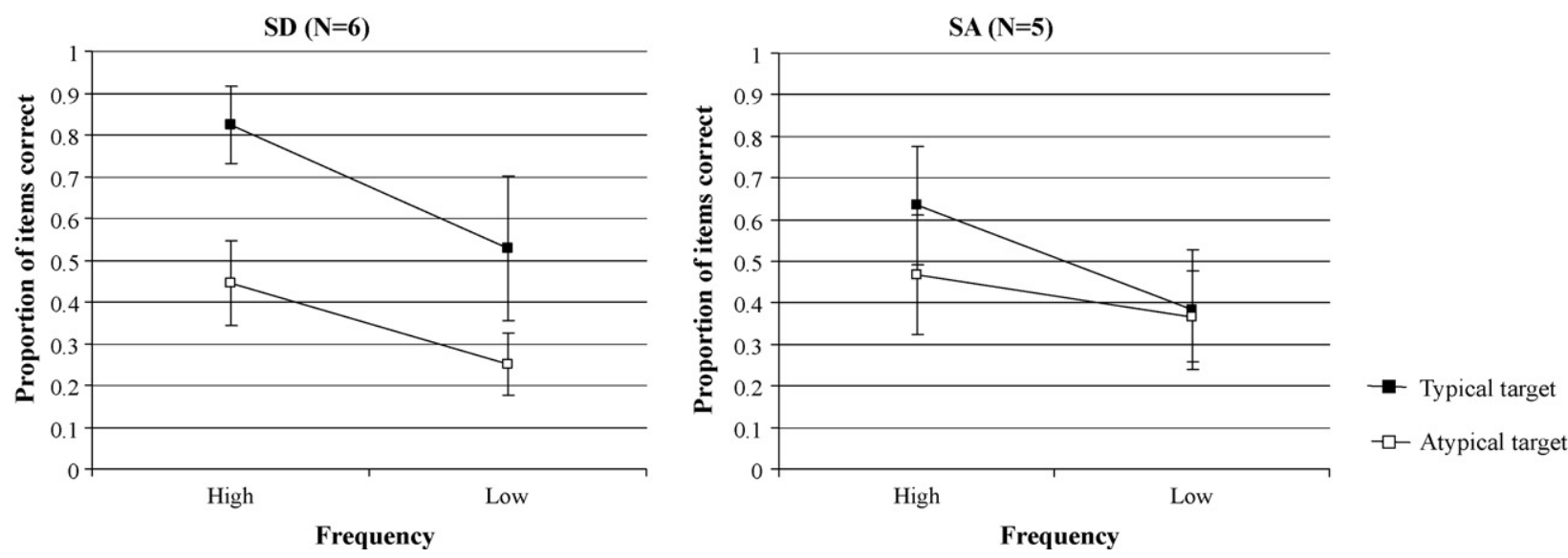

Fig. 5. Effect of frequency and regularity on spelling to dictation. 

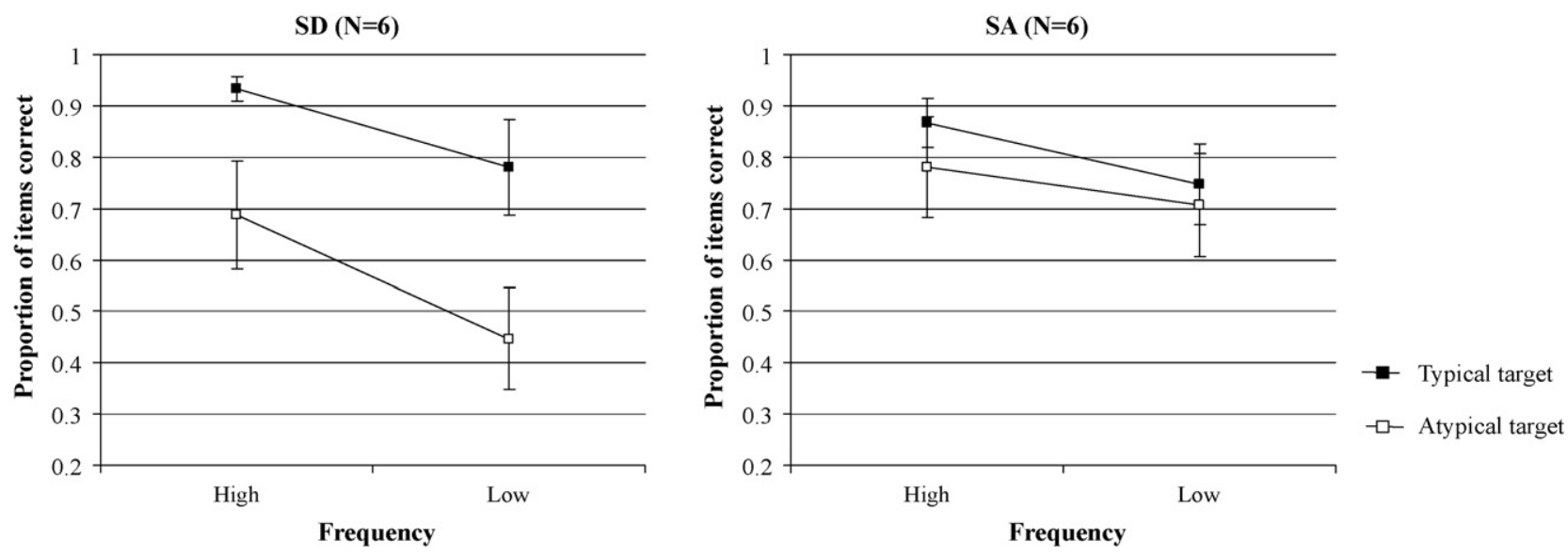

Fig. 6. Effect of frequency and regularity on past tense generation.

44\%; Fisher's exact one-sided $p<.04$ ), although the other patients did not. Patient NS scored $74 \%$ and $84 \%$ respectively for regular and irregular items, while PG, $\mathrm{CH}$ and ME were near ceiling on all conditions (PG: $94 \%$ vs. $86 \%$ for regular and irregular items; $\mathrm{CH}>96 \%$ for both; $\mathrm{ME}=90 \%$ for both ). In contrast, SD patients with a comparable degree of semantic impairment made substantial numbers of errors for low-frequency irregular items. A $2 \times 2 \times 2$ mixed factors ANOVA was used to contrast the effects of regularity and frequency in the two groups (see Fig. 6). This analysis included six SD patients with the mildest semantic deficits who showed the same degree of semantic impairment as the SA cases (comparison of word-picture matching and picture PPT for two subgroups: $t(10)<1)$. The interaction between patient group and regularity was significant: the SD patients showed a larger regularity effect than the SA cases, $F(1,10)=8.0, p=.02$. No other interactions reached significance. When considered alone, the SA group showed an effect of frequency, $F(1,5)=16.4, p=.03$, but no influence of regularity.

The SA patients' errors on the past tense generation task were again different from those seen in SD. The SA patients made frequent semantic and unrelated responses that were rarely seen in SD (an average of $13 \%$ and $10 \%$ of errors respectively). Semantic errors were typically past tense verbs with a similar meaning to the target verb (e.g., Today I drink wine, yesterday I supped wine). Many of the unrelated responses were also verbs produced in the past tense (e.g., Today I get hungry, yesterday I envied). Relatively few of the SA patients' errors were regularisations and LARC responses (an average of $7 \%$ and $3 \%$ of errors respectively) although every case produced some responses of this type. No change errors were also common (e.g., Today I play, yesterday I play; an average of $28 \%$ of errors). This error type was also observed quite commonly for the SD patients. There were small numbers of phonological, omission and grammatical errors (especially for patient SC; e.g., Today I drink wine, yesterday I drinking).

\subsection{Picture copying}

Illustrative pictures from the two patient groups are shown in Fig. 7. Five SA patients who were relatively good at reproducing pictures with no delay (77-85\% of features correct) completed the delayed picture copying task (64-70\% of features correctly repro- (a) Kangaroo

SD

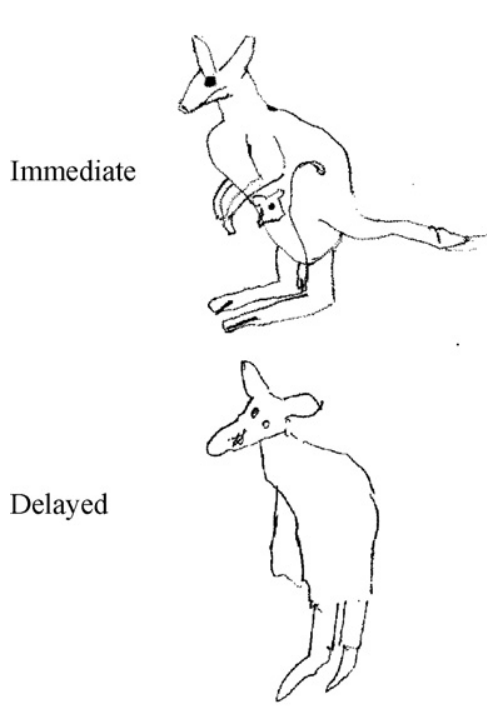

SA

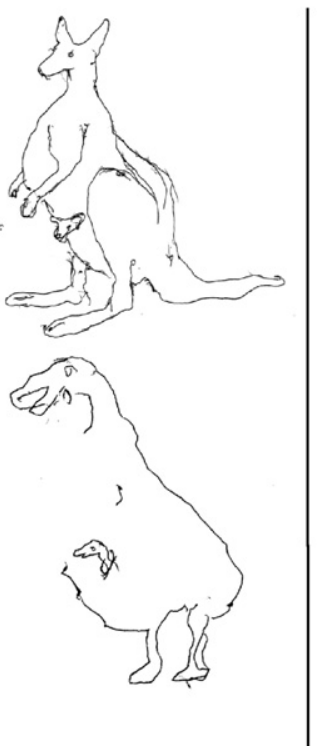

SD
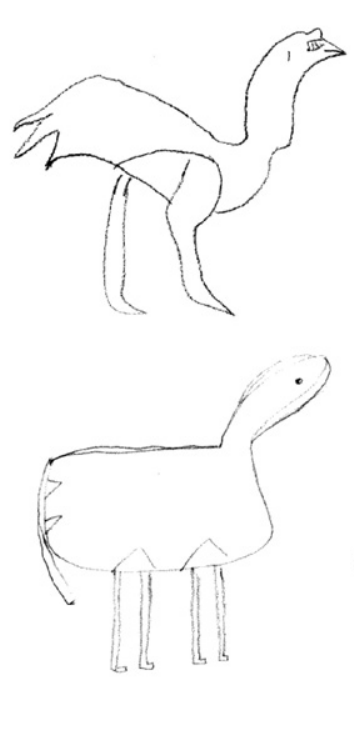

SA
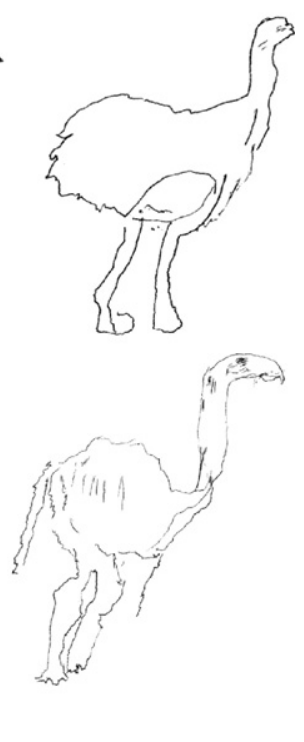

Fig. 7. Illustrative examples of immediate and delayed picture copying in semantic dementia and semantic aphasia. 
(a) Misses

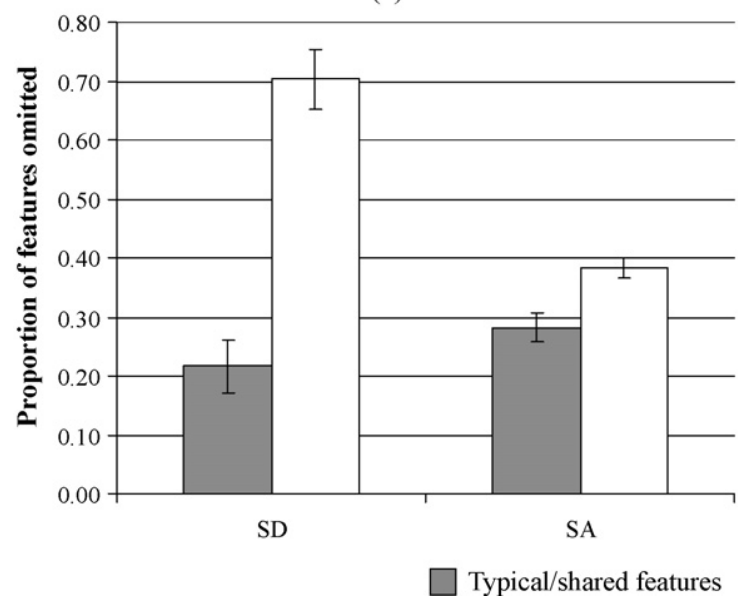

(b) Intrusions

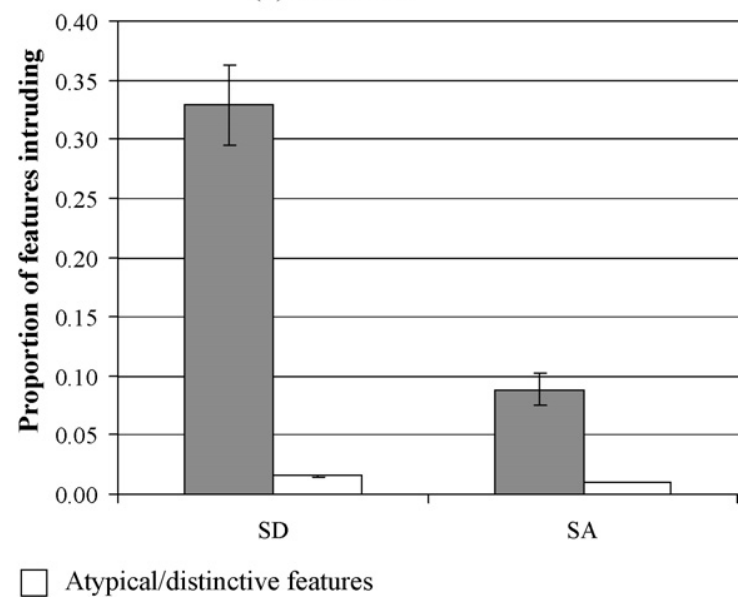

Fig. 8. Misses and intrusions of shared and distinctive features in delayed picture copying.

duced). These cases included individuals with both milder and more severe semantic deficits: as a group, their performance on semantic tasks was comparable to the first SD cohort (comparison of word-picture matching and picture PPT: $t(10)<1.5$, n.s.).

Following Patterson et al. (2006), we combined the shared within domain and shared within category elements to contrast shared vs. distinctive features. We performed two separate $2 \times 2$ mixed factors ANOVAs, examining the effects of patient group (SA: $N=5$; SD: $N=14$ ) and typicality/distinctiveness on (i) misses of correct features and (ii) intrusions of incorrect ones. Critically, for both measures, there was a distinctiveness by patient group interaction; misses: $F(1,17)=37.3, p<.0001$; intrusions: $F(1,17)=16.5, p=.0001$. Although the effect of typicality/distinctiveness was significantly larger for the SD than the SA group, the SA patients did show significant effects of distinctiveness for both misses, $t(4)=2.7, p=.05$, and intrusions, $t(4)=5.3, p=.006$. There was no main effect of group for feature omissions, indicating that the SD and SA patients reproduced equal numbers of correct features, $F(1,17)=2.9, p=.11$. However, the SD patients made more frequent intrusions than the SA patients overall, $F(1,17)=17.8, p=.001$. These data are shown in Fig. 8.

In addition, we examined contour violations for the six SA patients and the three SD patients who were tested both with and without a delay (see Fig. 9). A $2 \times 2$ mixed factors ANOVA was used to compare the effects of delay for these SA and SD groups. There was a significant main effect of delay indicating

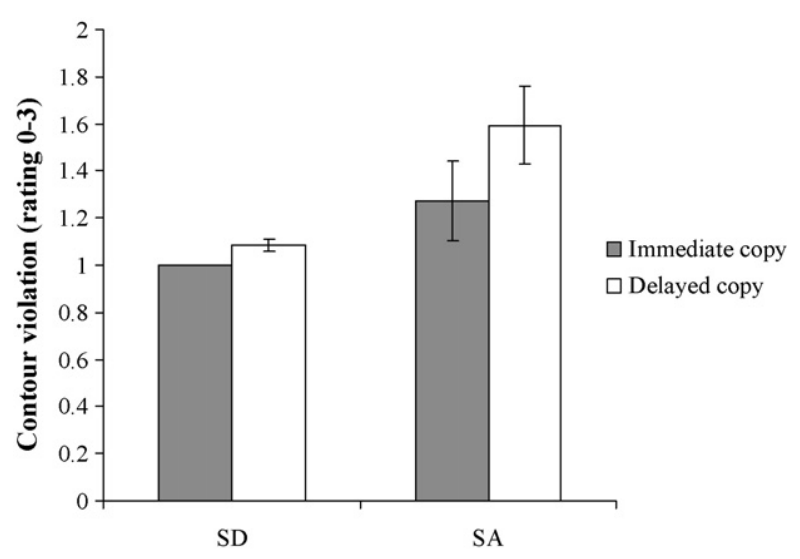

Fig. 9. Contour violations in immediate and delayed picture copying. Error bars show standard error of mean. that there were more contour violations in the delayed-copy condition $(F(1,6)=21.3, p=.004)$. There was also a significant interaction between patient group and delay, despite the small number of cases in the analysis $(F(1,6)=7.5, p=.03)$. The SA group produced more contour violations than the SD patients in delayed copying (Mann-Whitney $U=0, p=.03$ ) but not immediate copying (Mann-Whitney $U=3, n s$ ).

\subsection{Summary}

In all seven tasks, ANOVA yielded significant interactions between regularity/typicality and patient group: the SA patients were less sensitive to regularity/typicality than the SD patients, despite the fact that the two groups achieved equivalent scores on semantic tests. They also made different types of errors. The SD patients made predominately regularisation/LARC errors in every task requiring overt production-e.g., reading aloud (PINT to rhyme with 'mint'), spelling ('cough' $\rightarrow$ COFF), past tense generation ('catch' $\rightarrow$ 'catched') and delayed copying (drawing a duck with four legs). Although the SA patients did make errors of this type, the majority of their errors were implausible renderings of the stimuli. In reading aloud, the patients with better performance made largely implausible phonological errors while the more impaired patients made semantic/derivational errors, perseverations and omissions. In spelling, the patients made frequent letter substitutions, deletions and migrations. In the verb generation task, the SA patients responded with semantically related/unrelated verbs. In picture copying, the SA patients made a larger number of contour violations-for example, they sometimes omitted major features such as the heads of animals which did not make their drawings more typical.

\section{Discussion}

This study examined the impact of semantic impairment on a variety of "pre-semantic tasks" - lexical decision, object decision, colour decision, reading aloud, spelling to dictation, past tense generation and delayed picture copying - in semantic dementia (SD) and semantic aphasia (SA). Previous research indicates that all of these tasks are affected in a highly predictable way by the semantic degradation in SD (see Patterson et al., 2006). SD patients have difficulty with transformations that are atypical and make "regularisation errors" in which irregular items are produced as if they were domain-typical. In contrast, we found that comprehension impairment in SA did not result in this pattern. (1) In all 
seven "pre-semantic" tasks that we examined, the SA patients were less sensitive to typicality than the SD patients (producing significant group by regularity interactions), even though the two groups were matched for performance on semantic tests. (2) In several tasks, the aphasic group were also less sensitive to item frequency and/or showed a smaller frequency by typicality interaction than the patients with SD. (3) In production tasks, the SA patients made fewer regularisation errors and many more implausible responses than the SD group-for example, unrelated, semantic and perseverative errors.

We propose that these differences between SA and SD patients reflect the underlying nature of the semantic deficit in the two conditions: SD patients have degraded semantic representations, following atrophy of anterior temporal regions, while SA patients have deficient executive control over activation within the semantic system, following infarcts in left inferior frontal and temporoparietal regions (Jefferies \& Lambon Ralph, 2006). By this view, semantic degradation in SD directly affects the extent to which the semantic system can constrain the transformation in each "pre-semantic" task (for example, the transformation from orthography-to-phonology in reading aloud). The semantic degradation in SD has little impact on regular items, which are supported sufficiently by domain-specific representations alone. However, for low-frequency atypical items, regularisation errors occur because domain-specific representations are insufficient to generate the correct response in the absence of support from semantic memory (see Patterson et al., 2006). In contrast, for patients with SA, pre-semantic tasks should still be able to activate semantic representations in the undamaged ATL. Although the flow of activation through the network may be abnormal, atypical items will continue to receive support from the ATL and, as a consequence, we would not expect SA patients to show typicality effects to the same degree as individuals with SD. This key prediction was borne out on every task in this study. Moreover, the SA patients' errors were consistent with the hypothesis that poor executive control underpinned their deficits. The more severely impaired patients showed marked perseverations in several tasks (reading, past tense generation, and picture copying). In addition, the presence of semantic errors in past tense generation/reading and the misordering of letters in spelling to dictation might have reflected the patients' inability to overcome the activation of competitors.

Our proposed explanation of these 'pre-semantic' task differences in SD and SA fits well with the focus of cortical damage in each group. While the bilateral temporal poles, atrophied in SD, are thought to form a 'semantic hub' of amodal conceptual knowledge (Patterson, Nestor, \& Rogers, 2007; Visser et al., in press), the left prefrontal and temporoparietal areas, damaged in SA, are thought to contribute to semantic control processes, including controlled semantic retrieval and selection. Functional neuroimaging studies of healthy participants have particularly highlighted the role of the left inferior prefrontal gyrus in semantic control-however, posterior temporal and inferior parietal regions can also show sensitivity to the executive demands of semantic tasks (Badre, Poldrack, Pare-Blagoev, Insler, \& Wagner, 2005; Thompson-Schill et al., 1997; Wagner et al., 2001). These findings, together with our earlier observations of comparable semantic control deficits in SA following damage to either left prefrontal or temporoparietal cortex, suggest that semantic control is dependant on interactions between prefrontal and temporoparietal areas (Jefferies et al., 2007; Jefferies \& Lambon Ralph, 2006; Jefferies et al., 2008; Noonan et al., in press). Similarly, both prefrontal and parietal areas are recruited when healthy subjects perform executively demanding tasks beyond the semantic domain (Collette, Hogge, Salmon, \& Van der Linden, 2006; Collette et al., 2005; Garavan, Ross, Li, \& Stein, 2000; Nagel, Schumacher, Goebel, \& D’Esposito, 2008; Rowe,
Hughes, Eckstein, \& Owen, 2008) and damage to these two regions can result in comparable deficits in attention (Peers et al., 2005). This partial overlap in the neural basis of semantic control and executive control presumably explains the association between semantic impairment and poor performance on standard executive tasks in SA (Jefferies \& Lambon Ralph, 2006; Wiener et al., 2004).

To date, there has been relatively little consideration of the effect of poor executive control on "pre-semantic" tasks. Nevertheless, a recent study examined the impact of attentional deficits on past tense generation in Alzheimer's disease (AD) (Cortese et al., 2006). Patients with AD have attentional/executive deficits that are likely to contribute to their poor performance on semantic tasks (Baddeley, Baddeley, Bucks, \& Wilcock, 2001; Perry \& Hodges, 1999; Swanberg, Tractenberg, Mohs, Thal, \& Cummings, 2004). In line with this view, they are sensitive to the requirement for semantic selection/control (Nebes, Boller, \& Holland, 1986; Nebes, Brady, \& Huff, 1989; Papagno, Lucchelli, Muggia, \& Rizzo, 2003; Tippett, Gendall, Farah, \& Thompson-Schill, 2004). These patients also show evidence of a degraded store of semantic knowledge (Hodges, Patterson, Graham, \& Dawson, 1996; Hodges, Salmon, \& Butters, 1992) and they have temporal lobe atrophy that overlaps to some extent with the areas affected in SD (Galton et al., 2001). Patients with AD may therefore present with a combination of semantic degradation and deregulation. Cortese et al. (2006) found that both SD and AD patients showed effects of regularity and made regularisation errors in past tense generation. However, the effect of regularity/consistency was larger in SD, presumably because the SD group had more degraded semantic representations (the AD group were less impaired on semantic tasks). Strain et al. (1998) similarly found that regularity effects in AD patients' reading only emerged when the semantic impairment had become pronounced. Cortese et al. (2006) suggested that attentional deficits might produce an effect of regularity in past tense generation because multiple forms will be activated and a particular response must be selected for output. For example, executively impaired patients might make regularisation errors when generating the past tense of an irregular word like 'flee' if they select a highly activated but incorrect form such as 'fleed'. This might explain why the SA patients in our study produced some regularisation errors and showed small but significant effects of regularity on accuracy. However, it seems likely that degradation of the ATL semantic system also contributed to the regularity effects observed in AD by Cortese et al. (2006).

The current study provides much more information about how the relationship between semantic impairment and "presemantic" deficits is influenced by (i) the aetiology of brain damage (SD vs. SA) and (ii) the nature of the semantic impairment (degraded semantic representation in SD vs. poor executive control over semantic cognition in SA). The predicted effects of semantic impairment on pre-semantic tasks have been demonstrated most frequently in patients with SD. In contrast, patients who show a breakdown in this association - i.e., preserved reading of irregular words despite pronounced semantic memory problems - are typically individuals with $\mathrm{AD}$ and stroke aphasia (Gerhand, 2001; Lambon Ralph et al., 1995). These cases are viewed as highly significant by some researchers because they suggest that semantic memory impairment and regularisation errors on "pre-semantic" tasks are dissociable. However, our findings indicate that strong typicality effects in "pre-semantic" tasks follow degradation of ATL semantic representations and not poor executive control of semantic cognition. As the semantic deficit in $\mathrm{SA}$ and to some extent $\mathrm{AD}$ is likely to be related to poor executive control, individuals with these conditions do not disconfirm the hypothesis that semantic representations play an important role in "pre-semantic" cognition. 


\section{Acknowledgements}

We are indebted to the patients and their carers for their generous assistance with this study. We would like to thank Stephen Welbourne and Karalyn Patterson for useful discussions about these data. Thanks are also due to Karalyn Patterson for making the Cambridge SD data available for re-analysis and to Rachel Byrne, Linda Collier and Claire Slinger for referring some of the stroke aphasic patients to us. We gratefully acknowledge the help of Stephen Baker, Faye Corbett and Sheeba Ehsan with data collection and coding. The work was supported by grants from the NIMH (MH64445) and MRC (G0501632) and an RCUK fellowship awarded to E. Jefferies.

\section{References}

Baddeley, A. D., Baddeley, H. A., Bucks, R. S., \& Wilcock, G. K. (2001). Attentional control in Alzheimer's disease. Brain, 124, 1492-1508.

Badre, D., Poldrack, R. A., Pare-Blagoev, E. J., Insler, R. Z., \& Wagner, A. D. (2005) Dissociable controlled retrieval and generalised selection mechanisms in ventrolateral prefrontal cortex. Neuron, 47, 907-918.

Baldo, J. V., Dronkers, N. F., Wilkins, D., Ludy, C., Raskin, P., \& Kim, J. Y. (2005) Is problem solving dependent on language? Brain and Language, 92, 240250

Behrmann, M., \& Bub, D. (1992). Surface dyslexia and dysgraphia: Dual routes, single lexicon. Cognitive Neuropsychology, 9, 209-251.

Berthier, M. L. (2001). Unexpected brain-language relationships in aphasia: Evidence from transcortical sensory aphasia associated with frontal lobe lesions. Aphasiology, 15, 99-130.

Blazely, A. M., Coltheart, M., \& Casey, B. J. (2005). Semantic impairment with and without surface dyslexia: Implications for models of reading. Cognitive $\mathrm{Neu}$ ropsychology, 22, 695-717.

Borden, N. M. (2006). 3D angiographic atlas of neurovascular anatomy and pathology. Cambridge: Cambridge University Press.

Bozeat, S., Lambon Ralph, M. A., Graham, K. S., Patterson, K., Wilkin, H., Rowland, J., et al.(2003). A duck with four legs: Investigating the structure of conceptual knowledge using picture drawing in semantic dementia. Cognitive Neuropsychology, 20, 27-47.

Bozeat, S., Lambon Ralph, M. A., Patterson, K., Garrard, P., \& Hodges, J. R. (2000). Non-verbal semantic impairment in semantic dementia. Neuropsychologia, 38 , 1207-1215.

Breedin, S. D., Saffran, E. M., \& Coslett, H. B. (1994). Reversal of the concreteness effect in a patient with semantic dementia. Cognitive Neuropsychology, 11, 617660

Chertkow, H., Bub, D., Deaudon, C., \& Whitehead, V. (1997). On the status of object concepts in aphasia. Brain and Language, 58, 203-232.

Cipolotti, L., \& Warrington, E. K. (1995). Semantic memory and reading abilities: A case report. Journal of the International Neuropsychological Society, 1, 104-110.

Collette, F., Hogge, M., Salmon, E., \& Van der Linden, M. (2006). Exploration of the neural substrate of executive functioning by functional imaging. Neuroscience, 139, 209-221.

Collette, F., Van der Linden, M., Laureys, S., Delfiore, G., Degueldre, C., Luxen, A., et al. (2005). Exploring the unity and diversity of the neural substrates of executive functioning. Human Brain Mapping, 25, 409-423.

Coltheart, M., Byng, S., Masterson, J., Prior, M., \& Riddoch, M. J. (1983). Surface dyslexia. Quarterly Journal of Experimental Psychology, 35A, 469-495.

Conn, M. (2003). Neuroscience in medicine. Totowa, NJ: Humana Press.

Cortese, M. J., Balota, D. A., Sergent-Marshall, S. D., \& Buckner, R. L. (2003). Sublexical, lexical and semantic influences in spelling: Exploring the effects of age, Alzheimer's disease and primary semantic impairment. Neuropsychologia, 41, 952-967.

Cortese, M. J., Balota, D. A., Sergent-Marshall, S. D., Buckner, R. L., \& Gold, B. T. (2006). Consistency and regularity in past tense verb generation in healthy aging, Alzheimer's disease, and semantic dementia. Cognitive Neuropsychology, 23, 856-876.

Dilkina, K., McClelland, J. L., \& Plaut, D. C. (2008). A single-system account of semantic and lexical deficits in five semantic dementia patients. Cognitive Neuropsychology, 25, 136-164.

Funnell, E. (1995). Objects and properties: A study of the breakdown of semantic memory. Memory, 3, 497-518.

Funnell, E. (1996). Response biases in oral reading: An account of the co-occurrence of surface dyslexia and semantic dementia. Quarterly Journal of Experimental Psychology, 49A, 417-446.

Galton, C. J., Patterson, K., Graham, K., Lambon Ralph, M. A., Williams, G., Antoun, N. et al. (2001). Differing patterns of temporal atrophy in Alzheimer's disease and semantic dementia. Neurology, 57, 216-225.

Garavan, H., Ross, T. J., Li, S. J., \& Stein, E. A. (2000). A parametric manipulation of central executive functioning. Cerebral Cortex, 10, 585-592.

Gerhand, S. (2001). Routes to reading: A report of a non-semantic reader with equivalent performance on regular and exception words. Neuropsychologia, 39 1473-1484.
Gold, B. T., \& Buckner, R. L. (2002). Common prefrontal regions coactivate with dissociable posterior regions during controlled semantic and phonological tasks. Neuron, 34, 803-812.

Goodglass, H. (1983). The assessment of aphasia and related disorders (2nd ed.). Philadelphia: Lea \& Febiger.

Graham, N. L., Patterson, K., \& Hodges, J. R. (2000). The impact of semantic memory impairment on spelling: Evidence from semantic dementia. Neuropsychologia, $38,143-163$.

Hodges, J. R., Patterson, K., Graham, N., \& Dawson, K. (1996). Naming and knowing in dementia of Alzheimer's type. Brain and Language, 54, 302-325.

Hodges, J. R., Patterson, K., Oxbury, S., \& Funnell, E. (1992). Semantic dementia: Progressive fluent aphasia with temporal-lobe atrophy. Brain, 115, 17831806.

Hodges, J. R., Salmon, D. P., \& Butters, N. (1992). Semantic memory impairment in Alzheimer's disease: Failure of access or degraded knowledge. Neuropsychologia $30,301-314$

Hovius, M., Kellenbach, M. L., Graham, K. S., Hodges, J. R., \& Patterson, K. (2003). What does the object decision task measure? Reflections on the basis of evidence from semantic dementia. Neuropsychology, 17, 100-107.

Howard, D., \& Patterson, K. (1992). Pyramids and palm trees: A test of semantic access from pictures and words. Bury St. Edmunds, Suffolk: Thames Valley Test Company.

Jefferies, E., Baker, S. S., Doran, M., \& Lambon Ralph, M. A. (2007). Refractory effects in stroke aphasia: A consequence of poor semantic control. Neuropsychologia, $45,1065-1079$.

Jefferies, E., Jones, R., Bateman, D., \& Lambon Ralph, M. A. (2004). When does word meaning affect immediate serial recall in semantic dementia? Cognitive, Affective and Behavioral Neuroscience, 4, 20-42.

Jefferies, E., Jones, R. W., Bateman, D., \& Lambon Ralph, M. A. (2005). A semantic contribution to nonword recall? Evidence for intact phonological processes in semantic dementia. Cognitive Neuropsychology, 22, 183-212.

Jefferies, E., \& Lambon Ralph, M. A. (2006). Semantic impairment in stroke aphasia vs. semantic dementia: A case-series comparison. Brain, 129, 2132-2147.

Jefferies, E., Patterson, K., \& Lambon Ralph, M. A. (2008). Deficits of knowledge vs. executive control in semantic cognition: Insights from cued naming. Neuropsychologia, 46, 649-658.

Jefferies, E., Patterson, K., \& Lambon Ralph, M. A. (2009). Comprehension of concrete and abstract words in semantic dementia. Neuropsychology, 23, 492-499.

Joanisse, M. F. \& Seidenberg, M. S. (1999). Impairments in verb morphology afte brain injury: A connectionist model. Proceedings of the National Academy of Sciences of the United States of America, 96, 7592-7597.

Kay, J., Lesser, R., \& Coltheart, M. (1992). Psycholinguistic assessments of language processing in aphasia (PALPA). Hove, UK: Lawrence Erlbaum Associates.

Knott, R., Patterson, K., \& Hodges, J. R. (1997). Lexical and semantic binding effects in short-term memory: Evidence from semantic dementia. Cognitive Neuropsychology, 14, 1165-1216.

Knott, R., Patterson, K., \& Hodges, J. R. (2000). The role of speech production in auditory-verbal short-term memory: Evidence from progressive fluent aphasia. Neuropsychologia, 38, 125-142.

Lambon Ralph, M. A., Ellis, A. W., \& Franklin, S. (1995). Semantic loss without surface dyslexia. Neurocase, 1, 363-369.

Lambon Ralph, M. A., \& Howard, D. (2000). Gogi aphasia or semantic dementia? Simulating and assessing poor verbal comprehension in a case of progressive fluent aphasia. Cognitive Neuropsychology, 17, 437-465.

Marshall, J. C., \& Newcombe, F. (1973). Patterns of paralexia: A psycholinguistic approach. Journal of Psycholinguistic Research, 2, 175-199.

Moss, H. E., Tyler, L. K., Hodges, J. R., \& Patterson, K. (1995). Exploring the loss of semantic memory in semantic dementia: Evidence from a primed monitoring study. Neuropsychology, 9, 16-26.

Mummery, C. J., Patterson, K., Price, C. J., Ashburner, J., Frackowiak, R. S. J., \& Hodges, J. R. (2000). A voxel-based morphometry study of semantic dementia: Relationship between temporal lobe atrophy and semantic memory. Annals of Neurology 47, 36-45.

Nagel, I. E., Schumacher, E. H., Goebel, R., \& D'Esposito, M. (2008). Functional MRI investigation of verbal selection mechanisms in lateral prefrontal cortex. $\mathrm{Neu}$ rolmage, $43,801-807$.

Nebes, R. D., Boller, F. \& Holland, A. (1986). The use of semantic context by patients with Alzheimer's disease. Psychology and Aging, 1, 261-269.

Nebes, R. D., Brady, C. B., \& Huff, F. J. (1989). Automatic and attentional mechanisms of semantic priming in Alzheimers disease. Journal of Clinical and Experimental Neuropsychology, 11, 219-230.

Nestor, P. J., Fryer, T. D., \& Hodges, J. R. (2006). Declarative memory impairments in Alzheimer's disease and semantic dementia. Neuroimage, 30

Noonan, K., Jefferies, E., Corbett, F., Hopper, S., \& Lambon Ralph, M. A. (in press) Elucidating the nature of deregulated semantic cognition in semantic aphasia: Evidence for the roles of prefrontal and temporoparietal cortices. Journa of Cognitive Neuroscience.

Noppeney, U., Phillips, J., \& Price, C. (2004). The neural areas that control the retrieval and selection of semantics. Neuropsychologia, 42, 1269-1280.

Papagno, C., Lucchelli, F., Muggia, S., \& Rizzo, S. (2003). Idiom comprehension in Alzheimer's disease: The role of the central executive. Brain, 126, 24192430

Parkin, A. J. (1993). Progressive aphasia without dementia: A clinical and cognitive neuropsychological analysis. Brain and Language, 44, 201-220.

Patterson, K., \& Hodges, J. R. (1992). Deterioration of word meaning: Implications for reading. Neuropsychologia, 30, 1025-1040. 
Patterson, K., Lambon Ralph, M. A., Hodges, J. R., \& McClelland, J. L. (2001). Deficits in irregular past-tense verb morphology associated with degraded semantic knowledge. Neuropsychologia, 39, 709-724.

Patterson, K., Lambon Ralph, M. A., Jefferies, E., Woolams, A., Jones, R., Hodges, J., et al. (2006). 'Pre-semantic' cognition in semantic dementia: Six deficits in search of an explanation. Journal of Cognitive Neuroscience, 18, 169-183.

Patterson, K., Nestor, P. J., \& Rogers, T. T. (2007). Where do you know what you know? The representation of semantic knowledge in the human brain. Nature Reviews Neuroscience, 8, 976-987.

Peach, R. K. (2002). Treatment for phonological dyslexia targeting regularity effects. Aphasiology, 16, 779-789.

Peers, P. V., Ludwig, C. J. H., Rorden, C., Cusack, R., Bonfiglioli, C., Bundesen, C., et al (2005). Attentional functions of parietal and frontal cortex. Cerebral Cortex, 15 1469-1484.

Perry, R. J., \& Hodges, J. R. (1999). Attention and executive deficits in Alzheimer's disease: A critical review. Brain, 122, 383-404.

Plaut, D. C., McClelland, J. L., Seidenberg, M. S., \& Patterson, K. (1996). Understanding normal and impaired word reading: Computational principles in quasi-regular domains. Psychological Review, 103, 56-115.

Raven, J. C. (1962). Coloured progressive matrices sets $A, A B, B$. London: H.K. Lewis.

Robertson, I. H., Ward, T., Ridgeway, V., \& Nimmo-Smith, I. (1994). The test of everyday attention. Flempton: Thames Valley Test Company.

Rogers, T. T., Hodges, J. R., Lambon Ralph, M. A., \& Patterson, K. (2003). Object recognition under semantic impairment: The effects of conceptual regularities on perceptual decisions. Language and Cognitive Processes, 18, 625-662.

Rogers, T. T., Lambon Ralph, M. A., Garrard, P., Bozeat, S., McClelland, J. L., Hodges, J. R. et al. (2004). The structure and deterioration of semantic memory: A neuropsychological and computational investigation. Psychological Review, 111, 205-235.

Rogers, T. T., Lambon Ralph, M. A., Hodges, J. R., \& Patterson, K. (2004). Natural selection: The impact of semantic impairment on lexical and object decision. Cognitive Neuropsychology, 21, 331-352.

Rogers, T. T., Patterson, K., \& Graham, K. (2007). Colour knowledge in semantic dementia: It is not all black and white. Neuropsychologia, 45, 3285-3298.

Rowe, J., Hughes, L., Eckstein, D., \& Owen, A. M. (2008). Rule-selection and actionselection have a shared neuroanatomical basis in the human prefrontal and parietal cortex. Cerebral Cortex, 18, 2275-2285.

Saffran, E. M. (2000). The organization of semantic memory: In support of a distributed model. Brain and Language, 71, 204-212.
Snodgrass, J. G., \& Vanderwart, M. (1980). A standardized set of 260 pictures: Norms for name agreement, image agreement, familiarity and visual complexity. Journal of Experimental Psychology: Human Learning and Memory, 6, 174215.

Snowden, J. S., Goulding, P. J., \& Neary, D. (1989). Semantic dementia: A form of circumscribed cerebral atrophy. Behavioural Neurology, 2, 167-182.

Strain, E., Patterson, K., Graham, N., \& Hodges, J. R. (1998). Word reading in Alzheimer's disease: Cross-sectional and longitudinal analyses of response time and accuracy data. Neuropsychologia, 36, 115-171.

Swanberg, M. M., Tractenberg, R. E., Mohs, R., Thal, L. J., \& Cummings, J. L. (2004). Executive dysfunction in Alzheimer disease. Archives of Neurology, 61, 556-560.

Thompson-Schill, S. L., D’Esposito, M., Aguirre, G. K., \& Farah, M. J. (1997). Role of left inferior prefrontal cortex in retreival of semantic knowledge: A reevaluation. Proceedings of the National Academy of Sciences of the United States of America, 94, $14792-14797$.

Tippett, L. J., Gendall, A., Farah, M. J., \& Thompson-Schill, S. L. (2004). Selection ability in Alzheimer's disease: Investigation of a component of semantic processing. Neuropsychology, 18, 163-173.

Ullman, M. T., Corkin, S., Coppola, M., Hickok, G., Growdon, J. H., Koroshetz, W. J., et al. (1997). A neural dissociation within language: Evidence that the mental dictionary is part of declarative memory, and that grammatical rules are processed by the procedural system. Journal of Cognitive Neuroscience, 9, 266-276.

Visser, M., Jefferies, E., \& Lambon Ralph, M. A. (in press). Semantic processing in the anterior temporal lobes: A meta-analysis of the functional neuroimaging literature. Journal of Cognitive Neuroscience.

Wagner, A. D., Pare-Blagoev, E. J., Clark, J., \& Poldrack, R. A. (2001). Recovering meaning: Left prefrontal cortex guides controlled semantic retrieval. Neuron, 31, 329-338.

Warrington, E. K., \& James, M. (1991). The visual object and space perception battery. Bury St. Edmunds, Suffolk: Thames Valley Test Company.

Wechsler, D. (1987). Wechsler Memory Scale-Revised (WMS-R). New York: Psychological Corporation.

Wiener, D. A., Connor, L. T., \& Obler, L. K. (2004). Inhibition and auditory comprehension in Wernicke's aphasia. Aphasiology, 18, 599-609.

Woollams, A. M., Lambon Ralph, M. A., Plaut, D. C., \& Patterson, K. (2007). SDsquared: On the association between semantic dementia and surface dyslexia. Psychological Review, 114, 316-339. 